\title{
An Objective Identification and Climatology of Upper-Tropospheric Jets near Atlantic Tropical Cyclones
}

\author{
LEVI P. COWAN AND RoBERT E. HART \\ Department of Earth, Ocean, and Atmospheric Science, Florida State University, Tallahassee, Florida
}

(Manuscript received 8 August 2019, in final form 21 March 2020)

\begin{abstract}
An objective algorithm is developed for identifying jets in 200-hPa flow and applied to reanalysis data within $2000 \mathrm{~km}$ of Atlantic tropical cyclones (TCs) during 1979-2015. The resulting set of 16512 jets is analyzed both qualitatively and quantitatively to describe the climatology of TC-jet configurations and jet behavior near TCs. Jets occur most commonly poleward of TCs within the 500-1000-km annulus, where TC outflow amplifies the background potential vorticity gradient. A rigorous clustering analysis is performed, resulting in statistically distinct clusters of jet traces that correspond to common configurations of large-scale flow near Atlantic TCs. The speed structure of westerly jets poleward of TCs is found to vary with location in the Atlantic basin, but acceleration of jets downstream of their closest approach to the TC due to interaction with the TC's diabatic outflow is a consistent feature of these structures. In addition to the climatology developed here, this objectively constructed dataset of upper-tropospheric jets opens unique avenues for exploring TC-environment interactions and utilizing jets to quantitatively describe large-scale flow.
\end{abstract}

\section{Introduction}

Upper-tropospheric jets are among the most conspicuous environmental asymmetries that influence tropical cyclones (TCs), and have been argued to do so through a multitude of physical processes. While prior work has examined TC-jet interactions in case studies and modeling experiments, no systematic identification and cataloging of jets in proximity to TCs has been performed. Such a dataset would prove useful for analyzing specific TC-jet configurations and studying how TCs respond to nearby jets. In this study, jets near Atlantic TCs are objectively identified in 37 years of reanalysis data and examined both qualitatively and statistically.

\section{Summary of jet influences on TCs}

Prior work has demonstrated that jets can impact TC intensity and structure at multiple stages of evolution, from genesis and maturation to extratropical transition (ET). Jets may be detrimental to a TC by imparting vertical shear on the vortex (e.g., Park et al. 2009), but can interact constructively with a TC as well. Shi et al. (1990) showed in numerical experiments that jet streaks in the TC outflow layer generate transverse secondary

\footnotetext{
Corresponding author: Levi P. Cowan, levi.cowan@noaa.gov
}

circulations similar to those associated with midlatitude jet streaks (Uccellini and Kocin 1987). This was supported by later observations of Hurricane Florence (1988) (Shi et al. 1997). The ascending branch of the secondary circulation associated with a jet entrance region is located on the anticyclonic shear side of the jet, which relative to a nearby TC is often the radially inward side. For example, this is true of typical jet streaks poleward of a TC that are oriented eastward, in which the anticyclonic shear side of the jet faces the TC. This can allow dynamically forced ascent to superimpose onto the TC circulation, enhancing vortex-scale moist convection. This effect is magnified by the typically anticyclonic curvature of outflow jets (due to the radially outward component of TC outflow), which amplifies the ascending branch of the entrance region secondary circulation (Moore and Vanknowe 1992). Such forcing has been argued to have a causal link to the intensification of Hurricane Florence (1988) (Shi et al. 1997) and Hurricane Opal (1995) (Bosart et al. 2000). Case studies of ET events have also shown how nearby jet streaks can lead to deepening of the vortex both during and following the ET process (e.g., Klein et al. 2002; McTaggart-Cowan et al. 2003; Griffin and Bosart 2014).

Jets may also induce ascent near TCs through eddy fluxes of relative angular momentum. Molinari and Vollaro (1989) 
noted that the presence of an outward-directed outflow jet almost guarantees a net outward (inward) eddy transport of anticyclonic (cyclonic) relative angular momentum with respect to the vortex. These net eddy fluxes are usually largest in the outflow layer, where low inertial stability allows large asymmetries in the flow (Holland and Merrill 1984). From a balanced vortex perspective (Eliassen 1951), the resulting vertical gradient of cyclonic relative angular momentum import excites tropospheric ascent within the vortex (Ditchek et al. 2017), which may act constructively with ascent associated with the jet's ageostrophic secondary circulations and/or the TC convective core. It has been argued in several case studies that, by this mechanism, eddy relative angular momentum fluxes contributed to the intensification of real TCs (e.g., Molinari and Vollaro 1990; Molinari et al. 1998; Wu and Cheng 1999; Bosart et al. 2000).

Besides modulating the TC's secondary circulation directly, nearby jets have been demonstrated to provide low inertial stability channels through which mass is removed from the TC, thus reducing the work performed by the secondary circulation on the environment. Rappin et al. (2011) showed in a modeling experiment that introducing a westerly zonal jet poleward of a TC resulted in similar intensification rates and stronger peak intensity than an experiment with no jet present, and argued this was due to the low inertial stability channel provided by the jet. The jet experiment also exhibited a wavenumber 1 asymmetry in the TC core convection, possibly due at least partially to the aforementioned mechanisms of jet-forced ascent, along with the effects of shear (Corbosiero and Molinari 2002). This asymmetry appeared to aid the organization of the formative inner core compared to the no-jet experiment, which is consistent with other studies suggesting that upper-level environmental forcing plays a key role in TC genesis and early development (e.g., Pfeffer and Challa 1981; Bracken and Bosart 2000; Fischer et al. 2017). In addition, the idea of low inertial stability outflow channels being favorable for TCs is consistent with early observational work by Merrill (1988), wherein his intensifying composite consisted of more radially extensive, wider outflow jets than his nonintensifying composite.

These TC-jet interactions are highly coupled, since TCs also modulate the position, strength, and shape of nearby jets through the action of their diabatically driven outflow. For example, a westerly jet streak poleward of a TC may be strengthened by the confluence of outflow momentum into it and upper-level frontogenesis beneath it (Riemer and Jones 2010). This process can also be viewed as a tightening of the horizontal potential vorticity $(\mathrm{PV})$ gradient along the sloped tropopause
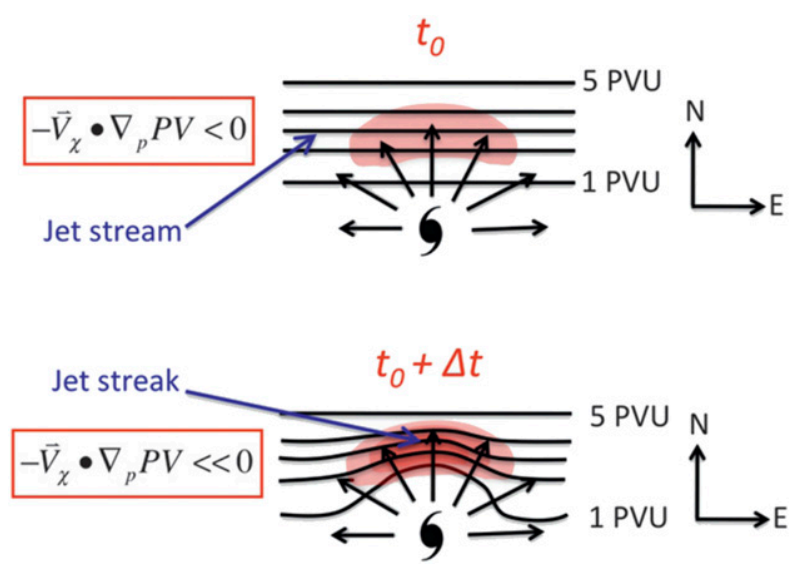

FIG. 1. Figure 4 from Archambault et al. (2013). Schematic of jet streak amplification associated with TC divergent outflow impinging upon an upper-tropospheric jet in the Northern Hemisphere. Vectors $\left(\mathbf{V}_{\chi}\right)$ denote the upper-tropospheric irrotational wind, contours the upper-tropospheric PV, and shading the advection of negative PV by the irrotational wind.

through radial advection of the TC's upper-level source of low PV (Fig. 1), or as the strengthening of a vorticity dipole formed by the TC's upper-level anticyclone and the cyclonic flow poleward of the jet (Cunningham and Keyser 2000; Pyle et al. 2004). Strengthening of the jet streak results in a stronger ageostrophic secondary circulation at the entrance region, which may enhance moist convection within the TC and therefore diabatic outflow, which in turn may strengthen the jet streak further. Thus, a coupled feedback can form between the jet and the TC (e.g., Bosart et al. 2000). However, this feedback is often strongly limited by the detrimental impacts of vertical shear on the TC implicit in the proximity of the jet. The evolution and consequences of these TC-jet interactions are therefore complicated and can be difficult to predict. In addition to modifying the TC itself, the impacts of such interactions on the environmental flow can propagate far from the TC along the midlatitude waveguide (e.g., Grams et al. 2011; Archambault et al. 2013).

With prior work (as discussed above) having demonstrated that interactions between TCs and uppertropospheric jets have important dynamical consequences, this motivates bulk observational analysis of jets near TCs to improve understanding and predictability of these interactions. A natural first step in such an analysis is to identify jets near TCs and examine the climatological distribution of TC-jet arrangements. As a quantitative analysis of this type has not been performed previously, this task is the primary goal of the present study. Herein, jets are objectively identified in reanalysis data near Atlantic TCs over a 37-yr period, 
forming a comprehensive dataset that is analyzed from a climatological perspective. Section 2 describes the data used, section 3 outlines the jet identification algorithm, sections 4 and 5 describe the climatology of the Atlantic TC-jet dataset, and section 6 provides an example of using this dataset to analyze jet speed structure climatology.

\section{Data}

Atlantic TC best track data are obtained from IBTrACS (Knapp et al. 2010) for the period 1979-2015. Only 6-hourly time steps corresponding to TCs of at least $35 \mathrm{kt}$ $\left(1 \mathrm{kt} \approx 0.51 \mathrm{~m} \mathrm{~s}^{-1}\right)$ intensity are selected. Time steps when the TC is subtropical or extratropical are removed, since we are interested in jets near cyclones that are primarily maintained by diabatic processes. Track points within $12 \mathrm{~h}$ of passing over landmasses are also removed. This leaves a total of 6934 samples from 409 TCs. ERAInterim reanalysis wind fields (Dee et al. 2011) are obtained for all of these TC samples and interpolated onto polar coordinate grids centered on each best track TC location. This is done by projecting the wind components onto an azimuthal equidistant projection (Snyder 1987) centered on the TC, which preserves all distances and angles relative to the origin, avoiding errors inherent in other transforms (Ahern and Cowan 2018). All resulting TC-centered grids extend to a radius of $3000 \mathrm{~km}$, and have radial and azimuthal grid spacings of $50 \mathrm{~km}$ and $2^{\circ}$, respectively. These values are chosen such that the radial spacing and maximum arclength between grid points is no larger than the ERA-Interim grid length of approximately $70 \mathrm{~km}$ within $2000 \mathrm{~km}$ of the TC, which is the radial domain focused on in this study.

\section{Jet identification}

An algorithm is developed here for objectively identifying jet axes in two-dimensional wind fields. For the purpose of identifying upper-tropospheric jets, wind fields on the $200 \mathrm{hPa}$ isobaric surface are used. This level is chosen as a compromise between the typical levels of polar jets (250-300 hPa), subtropical jets $(200 \mathrm{hPa})$, and TC outflow jets (150-200 hPa) (Koch et al. 2006; Ditchek et al. 2017). To facilitate the calculations used by this algorithm, TC-centered wind fields are interpolated onto a $50 \mathrm{~km}$ Cartesian grid. Because of the initial projection onto TC-centered azimuthal equidistant grids, distances and wind directions are preserved with minimal error.

A prior algorithm for identifying upper-level jet axes in two-dimensional flow was developed by Spensberger et al. (2017), which traces cross-flow wind speed maxima. Their approach is sensitive to higher-resolution datasets such as ERA-Interim, requiring significant filtering of the input fields to reduce noise and yield smoothly varying jet axes representative of synopticscale jet features. The resolution of the resulting piecewise curves representing jets is also limited by the grid spacing of the input data. Since smooth jet traces are important to the quality of various metrics derived from these traces, as well as quantitative comparisons of jets, we develop a different algorithm here that is less sensitive to the choice of input data and performs well for ERA-Interim, specifically.

Jets can be characterized to first order by local maxima in two-dimensional fields of wind magnitude in the cross-flow direction. Grid points are identified that satisfy the following conditions:

(i) $\exists \Delta n \in\{100,200,300,400,500 \mathrm{~km}\}\left(\left|\mathbf{v}_{n}\right|-\left|\mathbf{v}_{n+\Delta n}\right|\right.$ $\left.\geq 5 \mathrm{kt} \wedge\left|\mathbf{v}_{n}\right|-\left|\mathbf{v}_{n-\Delta n}\right| \geq 5 \mathrm{kt}\right)$,

(ii) $\neg \exists \Delta n \in\{100,200,300,400,500 \mathrm{~km}\}\left(\left|\mathbf{v}_{n}\right|-\left|\mathbf{v}_{n+\Delta n}\right|\right.$ $\left.<0 \wedge\left|\mathbf{v}_{n}\right|-\left|\mathbf{v}_{n-\Delta n}\right|<0\right)$

(iii) $\left|\mathbf{v}_{s}\right|-\left|\mathbf{v}_{s+\Delta s}\right|<10 \mathrm{kt} \vee\left|\mathbf{v}_{s}\right|-\left|\mathbf{v}_{s-\Delta s}\right|<10 \mathrm{kt}$,

(iv) $|\mathbf{v}| \geq 20 \mathrm{kt}$,

where $\mathbf{v}$ is the horizontal wind field, the subscript $n$ denotes values along the horizontal line normal to $\mathbf{v}, \Delta n$ is a characteristic half-width of upper-level jets, the subscript $s$ denotes values along the local streamline, and $\Delta s=250 \mathrm{~km}$. Condition (i) states that the wind speed at a point must be at least $5 \mathrm{kt}$ greater than at any pair of points spaced a distance $\Delta n$ on either side of the local streamline, where $\Delta n$ varies between 100 and $500 \mathrm{~km}$ in $100 \mathrm{~km}$ increments. This detects local maxima in the cross-flow direction. An illustration of these point pairs is given in Fig. 2, where the pair of green points would cause condition (i) to be satisfied at the blue point, since the blue point has a higher wind speed than both green points. Multiple values of $\Delta n$, and thus multiple pairs of points, are tested in order to capture jets of multiple widths in various scales of flow. The maximum value of $500 \mathrm{~km}$ for $\Delta n$ allows detection of jet maxima on width scales up to $1000 \mathrm{~km}$, which is large enough to encompass the vast majority of jet cores.

The second constraint (ii) disqualifies a grid point if the wind speed is less than at any of the same pairs of points tested in condition (i). This identifies situations where, even if a grid point is a local maximum in wind speed at some spatial scales [thereby satisfying (i)], it is a local minimum at other spatial scales. This usually indicates that two or more parallel jets are close together, but still distinct. Satisfying (i) and (ii) simultaneously allows such jets to be identified separately, provided they do not merge. An example can be seen in Fig. 2, 
which depicts two jets in close proximity. Given a value of $\Delta n$ that is sufficiently large (e.g., the green points), condition (i) would evaluate to true at points between the two jet axes (e.g., the blue square), since on large enough scales, these points appear as cross-flow maxima in wind speed. Therefore, in the absence of condition (ii), assuming all other conditions are satisfied, these points would be considered "jet-like." However, condition (ii) would evaluate to false between the jets, since those points are cross-flow minima in wind speed at some tested values of $\Delta n$ (e.g., the orange points). Thus, condition (ii) prevents the merging of two distinct cross-flow maxima, allowing the two jets to be identified separately.

An additional constraint is placed on how the wind speed varies along the local streamline. Condition (iii) states that the wind speed at a point cannot be $\geq 10 \mathrm{kt}$ less than at a point $250 \mathrm{~km}$ downstream and at a point $250 \mathrm{~km}$ upstream simultaneously. This serves to split a jet region in two when there is a small-scale, highamplitude local minimum in wind speed along the flow, indicating two jets separated by a small streamwise distance, such as is often the case across a sharp trough axis. Finally, the wind speed is required to be at least $20 \mathrm{kt}$ [condition (iv)]. Conditions (i), (ii), and (iv) are applied first, and then the boolean grid describing these conditions is smoothed with a $200 \mathrm{~km} \times$ $200 \mathrm{~km}$ boxcar kernel. This is done to improve continuity of regions satisfying these conditions. After the smoothing, condition (iii) is applied. For all points tested in conditions (i)-(iii), values are obtained through bilinear interpolation.

Regions are identified that satisfy all of these conditions. These are termed the "jet-like" regions of a given flow field. For each spatially distinct region, a set of test points along the cross-flow wind speed maximum (WSM) is selected. These are obtained by taking a set of uniformly distributed test points within the jet region (with a minimum separation of $100 \mathrm{~km}$ ) and displacing each in the direction normal to the flow that is toward increasing wind speed. This displacement stops when the wind speed gradient normal to the flow changes sign. If the region boundary is encountered before a sign change is found, the test point is discarded. From each of these points along the WSM, the local streamline is traced forward and backward until the boundary of the jet-like region is reached, forming candidate traces of the jet feature. The traces are allowed to exit and return to the same jet region if the distance traversed on the exterior is less than $500 \mathrm{~km}$. These traces are formed in $10 \mathrm{~km}$ increments, and at each step, the tracing trajectory is nudged slightly in the direction of the cross-flow wind speed gradient vector. This is necessary because jets do

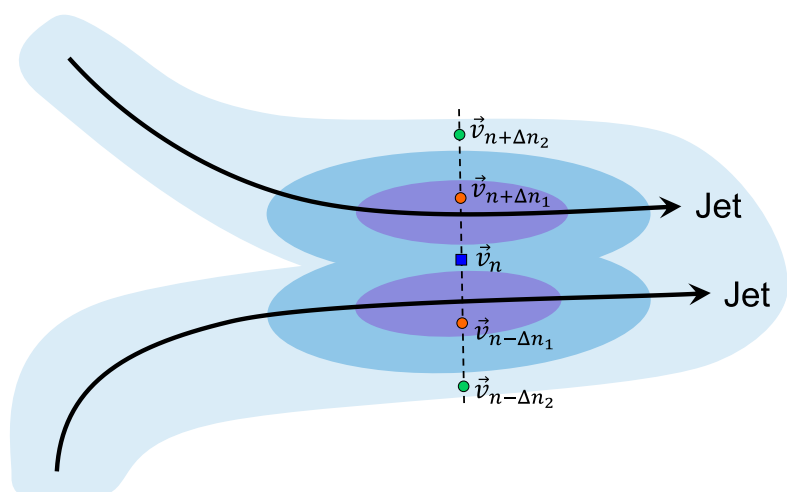

FIG. 2. Schematic depicting cross-flow points used in jet identification conditions (i) and (ii) for a case with two jets in close proximity. Shading denotes horizontal wind speed, increasing from light to dark colors. Jet axes are indicated by thick black arrows. The blue square denotes the grid point to evaluate, and $\mathbf{v}_{n}$ is the horizontal wind at that point. The black dashed line denotes the axis oriented normal to the local flow, and some example points spaced along this line are shown. The orange dots denote the pair of points spaced a distance $\Delta n_{1}$ from the grid point in the cross-flow direction, and the green dots are spaced a distance of $\Delta n_{2}$ from the grid point. The wind field evaluated at these point pairs $\left(\mathbf{v}_{n \pm \Delta n_{1}}\right.$ and $\mathbf{v}_{n \pm \Delta n_{2}}$ ) is used to evaluate conditions (i) and (ii). In a real use case for the identification algorithm, similar pairs of points are evaluated for all values of $\Delta n$ specified in conditions (i) and (ii).

not strictly follow isobaric streamlines, and while cores of high wind speed tend to align with the flow, tracing these cores accurately often requires departures from a single streamline. However, it is desirable to remain as close to a streamline as possible in order to accurately follow the jet flow direction. The nudging consists of adding a displacement $\mathbf{d}$ to the segment vector $\mathbf{s}$ defined by

$$
\mathbf{d}=A \tan \left(\theta_{\max }\right)|\mathbf{s}| \cdot \operatorname{sgn}(\nabla|\mathbf{v}| \cdot \hat{\mathbf{n}}) \cdot \hat{\mathbf{n}},
$$

where $\theta_{\max }$ is a maximum allowed angle of departure from the local streamline, $\hat{\mathbf{n}}$ is the horizontal unit vector normal to s, A is a scaling factor, and "sgn" denotes the sign function. Thus, $\mathbf{d}$ is perpendicular to $\mathbf{s}$, pointing toward higher wind speeds, and is scaled such that $\mathbf{d}$ is never larger than would be necessary to form an angle of $\theta_{\max }$ between $\mathbf{s}$ and $\mathbf{s}+\mathbf{d}$. An additional scaling factor $A$ is employed, defined as

$$
A=\min \left(\frac{|\nabla| \mathbf{v}|\cdot \hat{\mathbf{n}}|}{G}, 1\right)
$$

where $G$ is the value of the gradient below which to use scaling, here set to $1.03 \times 10^{-5} \mathrm{~s}^{-1}$ (equivalent to a change of $10 \mathrm{kt}$ over $500 \mathrm{~km}$ ). Thus, $A$ varies between 0 and 1 , and serves to limit nudging when the cross-flow wind speed gradient is nonzero but small, ensuring a smooth jet trace 
that asymptotes toward the jet core. Having defined the displacement, the nudged segment vector is then

$$
\mathbf{s}_{\text {nudged }}=|\mathbf{s}| \frac{\mathbf{s}+\mathbf{d}}{|\mathbf{s}+\mathbf{d}|},
$$

which preserves the segment's original length of $10 \mathrm{~km}$. Multiple values of $\theta_{\max }$ were tested, and qualitative analysis led to the selection of $20^{\circ}$ as the best balance between following the local streamline, following the jet core, and limiting sharp curvature. In all cases, the wind speed gradient is defined using centered finite differences with $\Delta x=\Delta y=100 \mathrm{~km}$.

Once all candidate jet traces have been computed with this procedure, one must be selected as the most representative of the jet feature. Only traces with a length of at least $1000 \mathrm{~km}$ are considered. The traces are compared by generating normalized scores of relevant attributes, given by

$$
q=\frac{X_{i}-\min \left\{X_{i}\right\}}{\max \left\{X_{i}\right\}-\min \left\{X_{i}\right\}},
$$

where $X$ is a given metric, the subscript $i$ denotes the $i$ th candidate jet trace, braces denote the set of values from all candidate traces, and $X$ is always defined such that larger values indicate a better score. Thus, the normalized score $q$ varies between 0 and 1 . Scores for each trace are generated from (4) for jet length (longer values score better) and the maximum crosswise distance from the nearest local WSM (smaller values score better). The trace chosen to represent the jet feature is the trace for which the sum of the values of $q$ for these two metrics is maximized.

Two examples of the jet traces output by this algorithm are shown in Fig. 3 for Hurricane Opal (1995) and Tropical Storm Allison (1995). Figure 3a is typical of the majority of cases in the dataset, where all jet features are successfully identified and closely follow the WSM. However, certain flow structures can result in some jets being missed by the algorithm. Figure $3 \mathrm{~b}$ is a case where two jets merge north of the TC, but are unable to be distinguished from each other, since the merged jet results in a single, continuous "jet-like" region. Thus, only the single jet trace within this region that scores best (here the northern jet) is recorded, and the jet closest to the TC goes unidentified. While complete misses of this type are rare in the dataset, merging or splitting jets generally pose difficulties for objective tracing, and are cases where inaccuracies are most likely to arise. Figure 3 also highlights examples of entrance region segments of jet streaks (traced in yellow). Entrance regions in this dataset are defined as continuous segments of a jet trace that meet an acceleration threshold of $+5 \mathrm{kt}$ over $200 \mathrm{~km}$, and are at least $200 \mathrm{~km}$ long. Segments meeting the acceleration threshold and separated by a distance shorter than $200 \mathrm{~km}$ are joined into one segment by including the intervening points.

Absent a previously accepted, quantitative definition of what constitutes a jet in two-dimensional flow, objectively evaluating the effectiveness of the jet identification algorithm is difficult. Extensive qualitative testing of the thresholds used in conditions (i)-(iii) was performed, with the final values tuned to yield the best agreement with how we might subjectively trace jet features. The tracing of jets that merge, are unusually wide, or are abnormally misaligned with the local streamlines is especially sensitive to the chosen values, and the algorithm can struggle in such edge cases. However, the successful identification of distinct, obvious jets is relatively insensitive to these choices, and the sensitivity is primarily associated with the aforementioned edge cases and weakly defined jets.

For detected jets, a reasonable test of a trace's quality is its proximity to the nearest local WSM in the cross-flow direction, used to approximate the location of the jet core. This metric is used explicitly in selecting the traces in the first place, but given that the tracer is bound to the local streamline within a tolerance, a trace never follows the WSM exactly, unlike the algorithm of Spensberger et al. (2017). Precisely following the WSM is often undesirable anyway, since the location of the gridpoint maximum can at times shift abruptly in regions of small-scale variability or fat jet cores, and allowing large curvature does not generally yield adequately smooth jet traces that represent the synoptic-scale jet axis. However, one would expect well-behaved jet traces to depart only mildly from the WSM at any point. Figure 4 a verifies that this is the case for jets near Atlantic TCs, showing that $99.9 \%$ of trace points are within $200 \mathrm{~km}$ of the WSM, and $89 \%$ are within one grid length of the ERA-Interim dataset (about $70 \mathrm{~km}$ ), which represents the smallest precision with which a WSM can be identified. Even for the largest departures from the WSM (Fig. 4b), 98\% remain within $200 \mathrm{~km}$, which the authors judge to be a reasonable maximum departure given the necessary constraints on the tracer.

This jet identification method is designed to be generally applicable (i.e., independent of flow characteristics and geographical region), but this climatological study focuses on jets near Atlantic TCs, specifically. Applying the tracing algorithm to the entire Atlantic TC dataset yields 30940 jets identified within $3000 \mathrm{~km}$ of $6933 \mathrm{TC}$ samples. A quality-control check then truncates jet traces that pass within $200 \mathrm{~km}$ of themselves, which trims jets that form repeating loops and removes jets that become trapped within a small area. This leaves a 

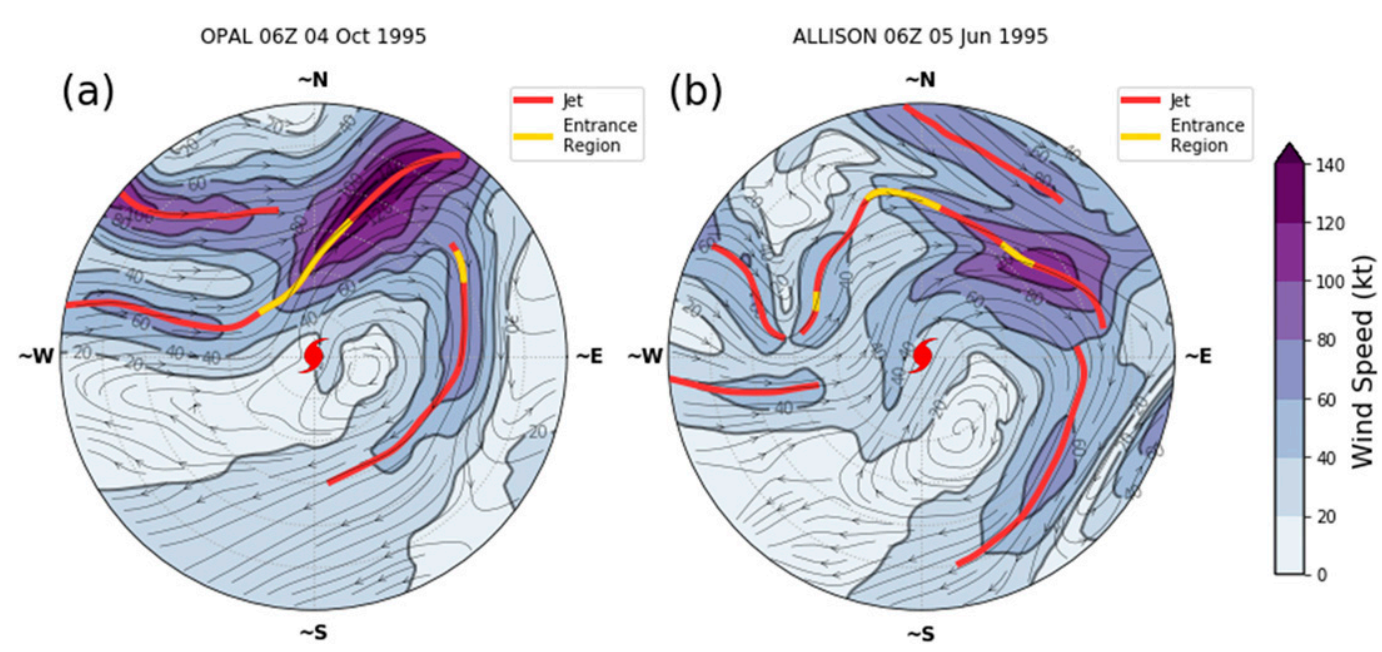

FIG. 3. $200 \mathrm{hPa}$ streamlines, isotachs (kt; shaded/contoured), and identified jet traces (red) within $3000 \mathrm{~km}$ of (a) Hurricane Opal at 0600 UTC 4 Oct 1995 and (b) Tropical Storm Allison at 0600 UTC 5 Jun 1995. The TC position at the origin is denoted by the hurricane symbol, and range rings are lightly dotted every $500 \mathrm{~km}$. Jet entrance regions are highlighted in yellow, as defined in the text. Panel (b) is a case where only one of two merging jets north of the TC is successfully identified. Note that the compass directions shown are everywhere approximate, except along the vertical line intersecting the origin.

dataset of 30932 jets from 6933 TC samples from 409 TCs. For the rest of this paper, analysis is restricted to $r \leq 2000 \mathrm{~km}$, unless stated otherwise. Clipping is done by deleting jet segments where $r>2000 \mathrm{~km}$ and retaining the remaining segments as separate jet features if they are longer than $1000 \mathrm{~km}$. This reduces the total number of jets to 16512 in 6864 samples from 409 TCs.

\section{General climatology characteristics}

The spatial frequency distribution of jets within a $2000 \mathrm{~km}$ radius is shown in Fig. 5a. Jets occur more commonly poleward than equatorward of the TC, as one would expect since most TCs form equatorward of the midlatitude waveguide. While jets are found to occur nearly everywhere around the TC, they are rare within about $700 \mathrm{~km}$ to the east of the TC. This domain minimum in frequency is attributable to the climatological position of the TC's outflow anticyclone, displaced east of the center by the background westerly vertical shear in the Atlantic basin. A corridor of most frequent jet occurrence is evident between 500 and $1000 \mathrm{~km}$ north of the TC, oriented west-southwest to east-northeast. This maximum denotes the zone where radial outflow tightens the background meridional PV gradient, leading to accelerating flow and the preferential generation of outflow jets on the poleward side of the TC. This is especially evident in the distribution of jet entrance regions (Fig. 5b), which is highly concentrated in the same region. This frequency maximum is closely paralleled by a nearby maximum in mean
PV frontogenesis by the irrotational wind at $200 \mathrm{hPa}$ (white contours in Fig. 5b), representing the aforementioned tightening of the PV gradient by the TC outflow. This maximum in jet and jet streak occurrence is the most pronounced feature of the climatological distribution of jets, and highlights the prevalence of interaction between Atlantic TCs and their upper-tropospheric environments.

Jets are nearly ubiquitous within $2000 \mathrm{~km}$ of Atlantic TCs, with at least one jet occurring within this distance in $99.9 \%$ of TC samples. In a large majority of cases $(81 \%)$, more than one jet exists within this distance (Fig. 6a). The most common number is two or three jets within $2000 \mathrm{~km}$, occurring in $68 \%$ of TC samples. Of particular interest are the jets that pass closest to TCs, and are thus most likely to interact directly with the TC outflow. The distribution of the distance of closest approach of jets nearest to all TC samples for which jets exist within $3000 \mathrm{~km}$ (6933 of 6934 samples) is shown in Fig. 6b. The mean distance of the nearest jet is $636 \mathrm{~km}$, which is close enough to interact directly with TC outflow, on average. An estimate of the radius at which TC outflow becomes dominantly rotational instead of radial is the Rossby radius of deformation, which has a median value of $673 \mathrm{~km}$ in the $150-250 \mathrm{hPa}$ layer within the $200-1000 \mathrm{~km}$ annulus using ERA-Interim fields. This is close to the radius at which the outward component of TC outflow begins decreasing with radius in ERAInterim, on average (Ditchek et al. 2017). The fact that the mean distance of the nearest jet is close to the Rossby radius indicates that jets are commonly close 
(a)

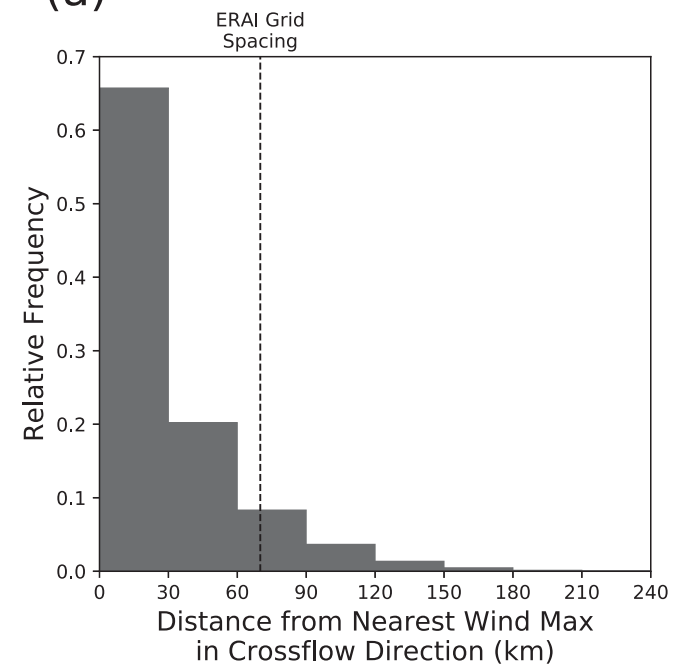

(b)

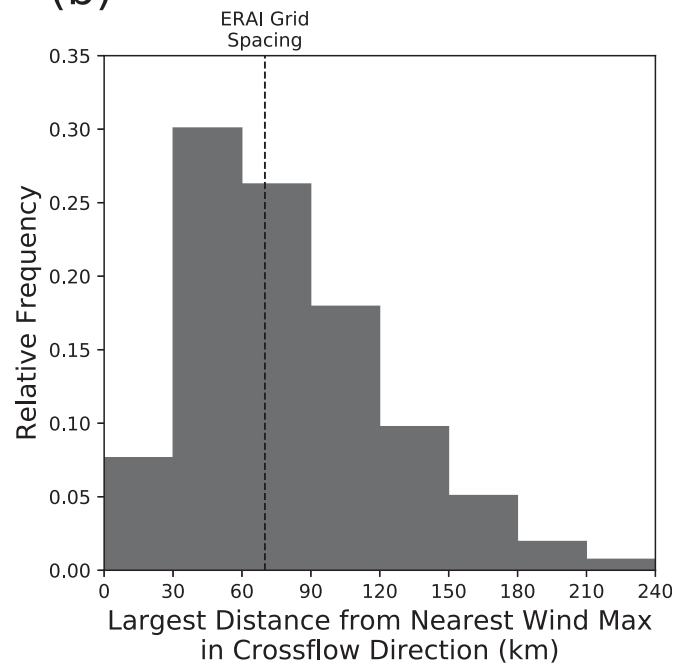

FIG. 4. Frequency distributions of the distance $(\mathrm{km})$ between jet traces and the nearest local WSM for (a) all jet trace points and (b) the most distant point from each jet trace. The nearest local WSM is defined as the point in the cross-flow, upgradient direction at which the wind speed gradient changes sign. The precision of this distance measurement is $10 \mathrm{~km}$. The dashed lines denote the approximate grid spacing of ERA-Interim (about $70 \mathrm{~km}$ ), which represents the smallest precision with which a WSM can be identified. These distributions are generated from 30935 jets identified in 6934 Atlantic TC samples.

enough to TCs to interact strongly with them through the processes outlined in section 1. It is also sensible that jets and their entrance regions are most common near the Rossby radius (Fig. 5), since it is at this radius that TC outflow begins to contribute significant geostrophic momentum to the environment, while simultaneously retaining a radial component that acts to cause upper-level frontogenesis. The roles of both of these processes in jet streak formation were described in Riemer and Jones (2010).

In some analyses that follow, it will be desirable to isolate the subset of jets typical of TC-environment interactions. As will be shown in section 5, the most common type of jet near Atlantic TCs is of generally

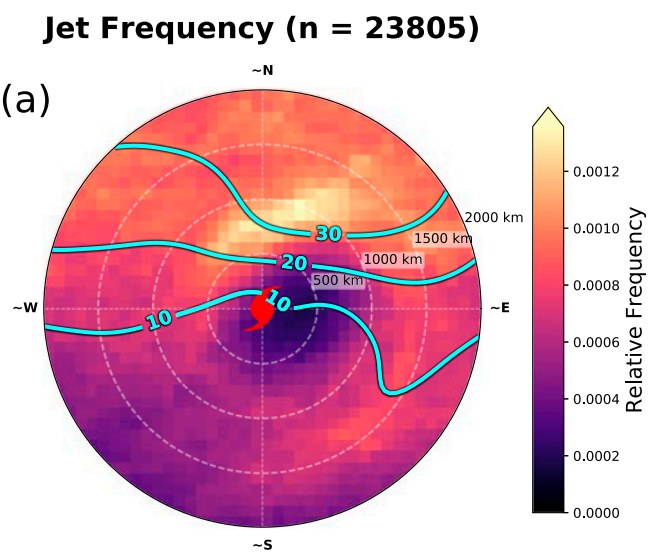

\section{Entrance Frequency $(n=18235)$}

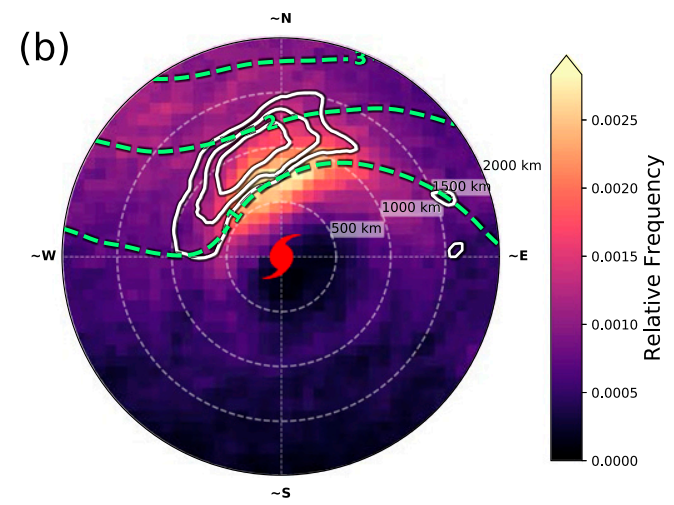

FIG. 5. Spatial relative frequency distribution of (a) jets and (b) entrance regions for the full jet dataset (shading). Data are binned by $100 \mathrm{~km} \times 100 \mathrm{~km}$ boxes, displayed on a polar coordinate system centered on the TC location, denoted by the hurricane symbol. The outer radius of the domain is $2000 \mathrm{~km}$, and the sample sizes of 23806 and 19537 , respectively, indicate the number of features intersecting the displayed domain. Bin counts are normalized by these sample sizes, such that the sums over the domains are equal to unity. In (a), mean 200-hPa wind speed from contributing samples is contoured every $10 \mathrm{kt}$. In (b), mean 200-hPa PV is contoured every 1 PVU in dashed green, and mean PV frontogenesis by the irrotational wind in solid white at contour levels of $0.4,0.6$, and 0.8 PVU $(1000 \mathrm{~km})^{-1} \mathrm{day}^{-1}$. Frontogenesis was computed after low-pass filtering the input fields for length scales of $200 \mathrm{~km}$. 
westerly orientation located poleward of the TC, broadly consistent with previous composites of TCenvironment interactions [e.g., Merrill (1988), Hanley et al. (2001), and Fischer et al. (2019)]. Some of these jets form within or interact with the TC outflow. We will call such jets "classic poleward jets" (CPJs). To identify CPJs, the jet with the nearest location of closest approach (LCA) to each TC sample occurring in the northern semicircle is selected, and only retained if it is anticyclonic shear-facing at the LCA, which means the side of the jet with anticyclonic flow is facing the TC at the LCA. Thus, when these criteria are met for a TC sample, only one CPJ can exist for that sample, since the nearest jet (in the northern semicircle) is chosen. This is done in the interest of isolating only the jets with which the TC is most likely to be interacting, since the nearest jet typically acts as an inertial barrier that blocks TC outflow from expanding beyond it and interacting with more distant jets. This can be seen in the mean radial profile of 200-hPa radial wind at the azimuth of the closest approach of the nearest jet in each TC sample (Fig. 7). Mean outflow occurs radially inward of the closest jet's LCA, but quickly decreases beyond the radius of the jet, reversing to mean inflow at about $200 \mathrm{~km}$ radially outward from the LCA. It is important to distinguish between two different types of CPJs: those which approach the TC from the environment (thus the LCA occurs in the middle of the jet), and those whose LCAs are at the beginning of the jet, which means they usually originate within the TC outflow. These will be termed "environmental CPJs" and "outflow CPJs," respectively. A total of 4391 environmental CPJs and 1700 outflow CPJs are identified that pass within $2000 \mathrm{~km}$ of a TC, together comprising $37 \%$ of the dataset within that radius. The spatial frequency distributions of these two sample sets are shown in Fig. 8. Their shapes and mean jet traces make the distinction between the two CPJ types clear, with environmental CPJs originating far upstream (west) of the TC on average, while outflow CPJs originate close to the TC and follow a typical outward, anticyclonically curving path.

\section{Jet clustering}

\section{a. Individual jets}

With a large jet dataset developed, a natural question is: What jet types are most common near TCs? To answer this question, a method of grouping jets with similar characteristics must be formulated. Necessary to any such analysis is a metric quantifying differences between jets. Let two jets be defined by the sets of points $P=$ $\left\{\mathbf{p}_{0}, \ldots, \mathbf{p}_{M}\right\}$ and $Q=\left\{\mathbf{q}_{0}, \ldots, \mathbf{q}_{N}\right\}$ containing $M$ and $N$ points, respectively, where $M \leq N$, each $\mathbf{p}_{i}$ and $\mathbf{q}_{j}$ is an $(r, \theta)$ position vector, and these points are ordered forward along the jet traces. One measure of dissimilarity between the jets is the mean Euclidean distance between them, evaluated for the segment of the longer jet (of same length as the shorter jet) where this value is minimized:

$$
\overline{\Delta x}=\frac{1}{M} \sum_{i=0}^{M} d\left(\mathbf{p}_{i}, \mathbf{q}_{i+k^{*}}\right),
$$

where

$$
\begin{array}{r}
k^{*}=\arg \min _{0 \leq k \leq N-M}\left[\frac{1}{M} \sum_{i=0}^{M} d\left(\mathbf{p}_{i}, \mathbf{q}_{i+k}\right)\right], \\
d(\mathbf{p}, \mathbf{q})=\sqrt{r_{p}^{2}+r_{q}^{2}-2 r_{p} r_{q} \cos \left(\theta_{p}-\theta_{q}\right)} .
\end{array}
$$

The mean absolute angle difference between the trace trajectories is also computed for the same jet segment:

$$
\overline{\Delta \phi}=\frac{1}{M} \sum_{i=1}^{M} \cos ^{-1} \frac{\left(\mathbf{p}_{i}-\mathbf{p}_{i-1}\right) \cdot\left(\mathbf{q}_{i+k^{*}}-\mathbf{q}_{i+k^{*}-1}\right)}{\left|\mathbf{p}_{i}-\mathbf{p}_{i-1}\right|\left|\mathbf{q}_{i+k^{*}}-\mathbf{q}_{i+k^{*}-1}\right|} .
$$

The final dissimilarity metric is a linear combination of these two component metrics:

$$
D=\alpha \overline{\Delta x}+\beta \overline{\Delta \phi},
$$

where $\alpha$ and $\beta$ are scaling factors. These are subjectively set to $\alpha=10^{-6} \mathrm{~m}^{-1}$ and $\beta=4 / \pi$, such that a $1000 \mathrm{~km}$ mean separation between two jets has a contribution to $D$ equal to that of a mean trajectory difference of $45^{\circ}$. After testing many combinations of these coefficients, these values were found to yield qualitatively coherent jet groups in the cluster analysis that follows. As one might expect, $\overline{\Delta x}$ and $\overline{\Delta \phi}$ are correlated, but only moderately (linear correlation of 0.28 ); thus, the parameter space spanned by them is large (not shown). This indicates the high frequency of jet pairs with similar (different) orientation but large (small) separation, thus necessitating the use of both parameters to define dissimilarity. Note that this dissimilarity metric includes no information about wind speed, and is purely a measure of differences between jet paths. As a sanity check for this choice of dissimilarity metric, the Fréchet distance (Eiter and Mannila 1994) was also tested (using equallength jet segments), which yielded similar clustering results to those that follow later in this section, with minor differences (not shown). The present metric was chosen due to its greater versatility, since it allows the 

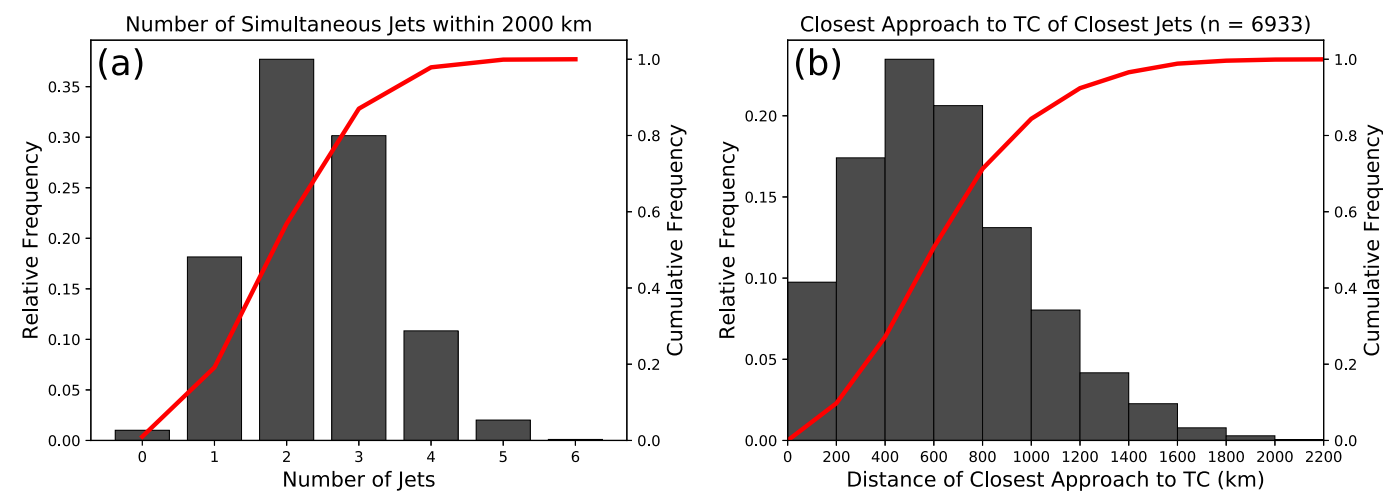

FIG. 6. Frequency distribution (gray histogram) and cumulative frequency distribution (red line) of (a) the number of jets simultaneously present within $2000 \mathrm{~km}$ of a TC sample and (b) distances of closest approach of the nearest jets to each TC sample.

relative contributions of distance and trajectory to jet dissimilarity to be modified, facilitating sensitivity testing of the resulting cluster characteristics.

To use this dissimilarity metric to group jets together, the Unweighted Pair Group Method with Arithmetic Mean (UPGMA) hierarchical agglomerative clustering algorithm (Sokal and Michener 1958; Sneath et al. 1973) is employed. This method is appropriate for a dataset in which the number of clusters is unknown, as we have here. However, an optimal number of clusters must still be determined. For agglomerative methods, this consists of choosing a level at which to truncate the cluster hierarchy. The information needed to make this choice is contained in the rate-distortion curve, which measures within-cluster dispersion as a function of the number of clusters. Here, the dispersion $W(m)$ associated with $m$ clusters is estimated by the distance between the two clusters combined in the merging step that reduces $m+1$ clusters to $m$ clusters within the hierarchy. Following Kolesnikov et al. (2015), a cost function is formulated by parameterizing the rate-distortion curve in the following way:

$$
\hat{W}(m)=K m^{-2 / a},
$$

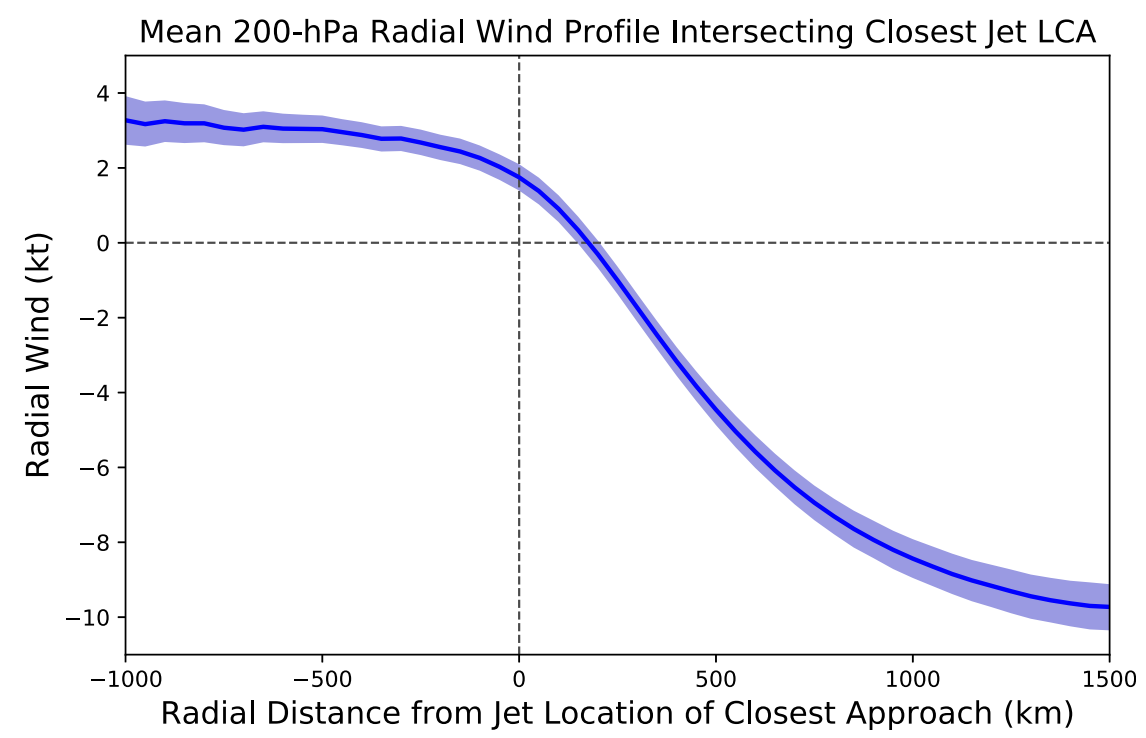

FIG. 7. Mean radial profile of 200-hPa radial wind at the azimuth of the LCA of the nearest jet to each TC sample. The profile is aligned relative to the jet LCA, such that the $x$ axis is radial distance from the LCA, extending from $1000 \mathrm{~km}$ radially inward of the LCA to $1500 \mathrm{~km}$ radially outward from the LCA. Sample sizes vary along the profile due to variance in LCA radius within the bounded domain $(r \in[0,3000] \mathrm{km})$. Shading denotes the $95 \%$ confidence interval about the mean from resampling with replacement 10000 times. 

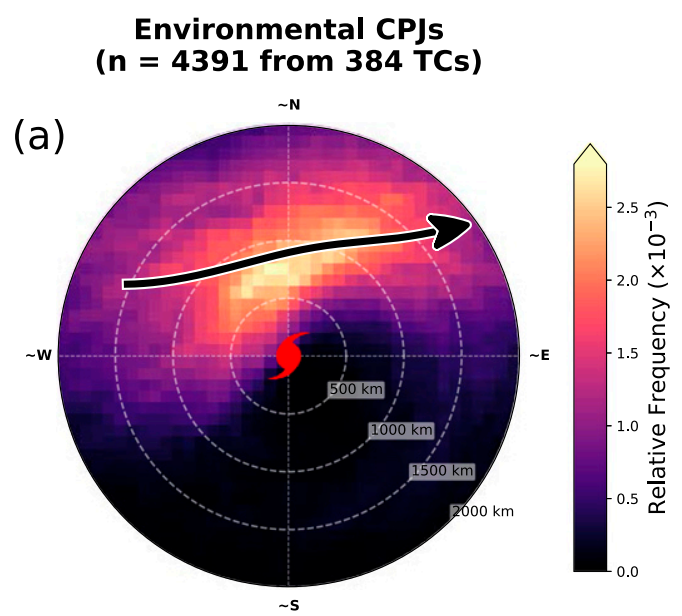

\section{Outflow CPJs ( $n=1700$ from $294 \mathrm{TCs}$ )}

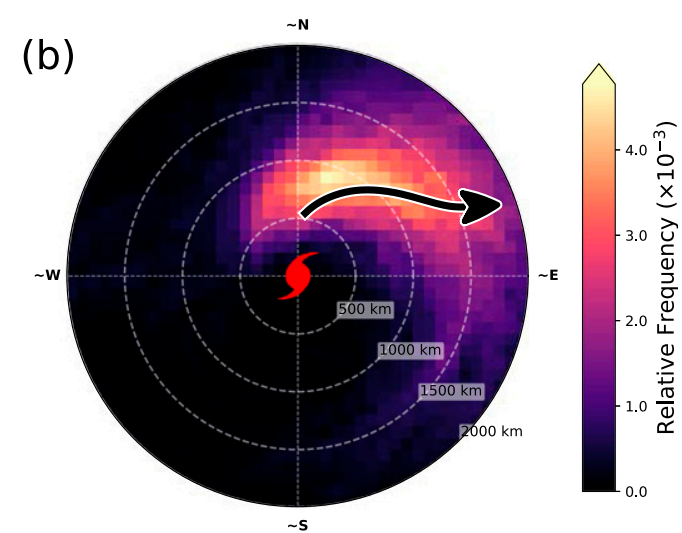

FIG. 8. Spatial relative frequency distribution of (a) environmental CPJs and (b) outflow CPJs. Data are binned by $100 \mathrm{~km} \times 100 \mathrm{~km}$ boxes, displayed on a polar coordinate system centered on the TC location, denoted by the hurricane symbol. The outer radius of the domain is $2000 \mathrm{~km}$, and the sample sizes of 4391 and 1700, respectively, indicate the number of jets intersecting the displayed domain. Bin counts are normalized by these sample sizes, such that the sums over the domains are equal to unity. Thick blue arrows denote the mean jet traces for both samples.

where $K$ is a constant and $a$ is a parameter that is found by fitting $W$ via least squares to the log-linear model:

$$
\log _{10} \hat{W}(m)+\frac{2}{a} \log _{10} m+b=0,
$$

where $b$ is a constant. With a suitable value of $a$ determined, $\hat{W}(m)$ approximates the rate-distortion curve that would theoretically result if the dataset was uniformly distributed with no clustering. A cost function can then be defined that measures departures from uniformity:

$$
C(m)=W(m) \cdot m^{2 / a} .
$$

Local minima in $C$ indicate values of $m$ for which the balance between quantization error and complexity of representation (number of clusters) is optimized. Equation (9) is fit over the domain $m \in[1,50]$ after testing the upper bound to ensure that the resulting cost function is stable and that relevant local minima occur within that interval.

It is important to note that it should not be assumed a priori that the data can be usefully clustered at all. If jets were distributed sufficiently uniformly according to the dissimilarity metric, clusters would simply represent arbitrary samples from the population with constrained variance, containing no structural information about the dataset. Since jet paths have high variance, we do not expect particularly strong clustered structure in this dataset. However, we do expect some jet types to be significantly more common than others, and to the extent that these statistical modes form clusterable structure, this analysis should identify such modes. Regardless of the degree of clustered structure, the results of clustering will be essential for describing the distribution of jets, by breaking jet axes into coherent groups and comparing the relative sizes of those groups.

The rate-distortion curve in Fig. 9a closely follows the model for uniform data, with only small departures relative to the range of values. However, inspection of the cost function in Fig. 9b reveals that, relative to the function's range on the interval $m \in[1,50]$, two distinct local minima do exist at $m=2$ and $m=8$. These suggest the possibility of efficient representations of the dataset with 2 or 8 clusters. While $m=2$ is the global minimum in the cost function on the interval $m \in[1,50]$, two clusters are considered too few to represent the jet distribution with sufficient precision. This demarcation is associated with the broad partitioning of jets between those northwest of the TC oriented generally northeastward and those southeast of the TC oriented generally southwestward (not shown). The cost function minimum at 2 clusters indicates a preference for these two general jet orientations, but cluster dispersion is large at $m=2$, which results in a very coarse representation of the distribution. Clipping the cluster hierarchy at $m=8$ is thus chosen as the optimal partitioning.

The jet clusters resulting from enforcing $m=8$ are shown in Fig. 10, their associated mean upper-tropospheric environments in Fig. 11, and their TC sample locations in Fig. 12. With the large sample-to-cluster ratio, there is strong dispersion in all clusters, but the clusters are visually distinct from one another in position and orientation relative to the TC (Fig. 10). Cluster 1 is the largest 

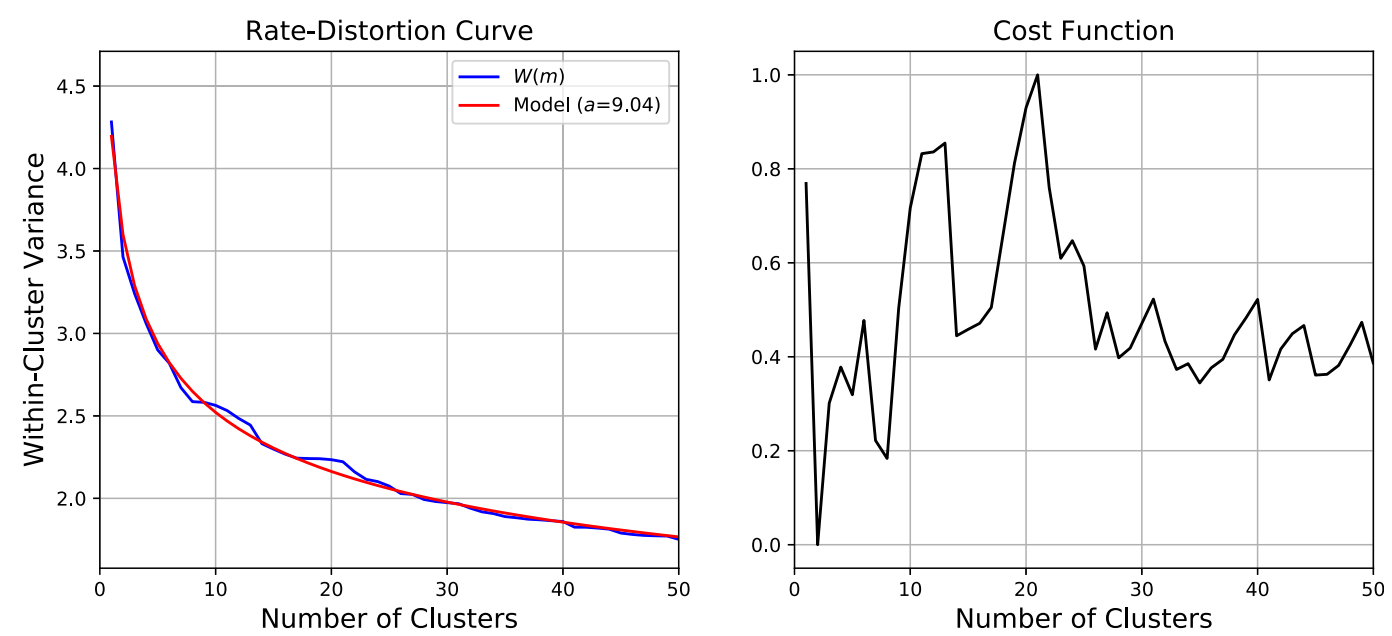

FIG. 9. (a) The rate-distortion curve $W(m)$ for the full jet dataset (blue) and the parameterized model with $a=9.04$ (red), both plotted over the domain $m \in[1,50]$. The model is fit over the same domain. (b) The cost function defined in (10) using $a=9.04$. For $m>50$, this function decreases nearly monotonically without significant local extrema.

by a significant margin, containing $44 \%$ of all jet samples, as it aggregates nearly all jets passing north of the TC oriented generally eastward. Such jets are common, occurring in $84 \%$ of TC samples (within the $2000 \mathrm{~km}$ radius limit considered here). The dominance of this type of jet is consistent with nearly all TC samples being located south of the climatological position of the polar jet (Fig. 12), as well as the tendency of the meridional PV gradient to become enhanced poleward of TCs, facilitating westerly jet formation.

Several of the remaining primary clusters are associated with common flow regimes near TCs. Clusters 2 and 5 contain anticyclonically curved outflow jets wrapping around the southern and eastern flanks of the TC, respectively, as well as any environmental jets with similar orientations. These are common when a TC is located beneath a zonally elongated $200 \mathrm{hPa}$ ridge axis (Fig. 11, Cluster 2) or a region of high PV exists to the east and deforms the outflow (Fig. 11, Cluster 5). This latter configuration is associated with TC samples located predominantly upstream of the climatological position of the midoceanic trough (Fig. 12, Cluster 5), where the probability of positive PV anomalies occurring east of the TC is maximized. Cluster 3 contains westerly jets to the south of TCs, associated with low-tropopause environments where the TC is north of the subtropical jet or beneath an upper-tropospheric trough. From Fig. 11, Cluster 3 has a mean $200 \mathrm{hPa}$ PV at the TC location of about 1.5 PVU (1 PVU $=10^{-6} \mathrm{~K} \mathrm{~kg}^{-1} \mathrm{~m}^{2} \mathrm{~s}^{-1}$ ), higher than any other cluster. Unsurprisingly, it also has the highest mean latitude $\left(31.5^{\circ} \mathrm{N}\right)$ by a significant margin. Cluster 4 contains northerly or northeasterly jets west of the TC, typically associated with anticyclonic wave breaking (AWB) events or otherwise amplified troughs immediately upstream of the TC. The two smallest clusters (6 and 7), together accounting for $5.5 \%$ of samples, are loosely associated with less common configurations of cyclonic wave breaking west of the TC (Cluster 6) and anticyclones north of the TC (Cluster 7). Characteristics and qualitative descriptions of the primary clusters are summarized in Table 1 .

\section{b. Group clustering}

Since more than one jet is present in $81 \%$ of TC samples for this particular radial domain (Fig. 6a), there is considerable overlap between the clusters of individual jets in TC sample space (hereafter referred to as "individual clusters"). For example, in Fig. 10, 88\% of the TC samples in Individual Cluster 2 are also present in Individual Cluster 1, indicating that jets from both clusters are often present simultaneously. Therefore, to assess the climatological distribution of TC-jet configurations, clustering of jet groups is performed.

Jet group datasets are formed by defining all jets in each TC sample as a group and then organizing the groups by size. There are 1259 groups of one jet from 268 TCs, 2615 groups of two jets from 365 TCs, 2091 groups of three jets from 339 TCs, and 899 groups of four or more jets from 221 TCs. Sets of TC samples with identical numbers of jets present are analyzed separately, since jet groups can only be reasonably compared (and thus clustered) if they are of equal size. A dissimilarity metric for two given jet groups is defined as follows:

1) All possible pairing arrangements between the two groups are formed. For each such arrangement, the 

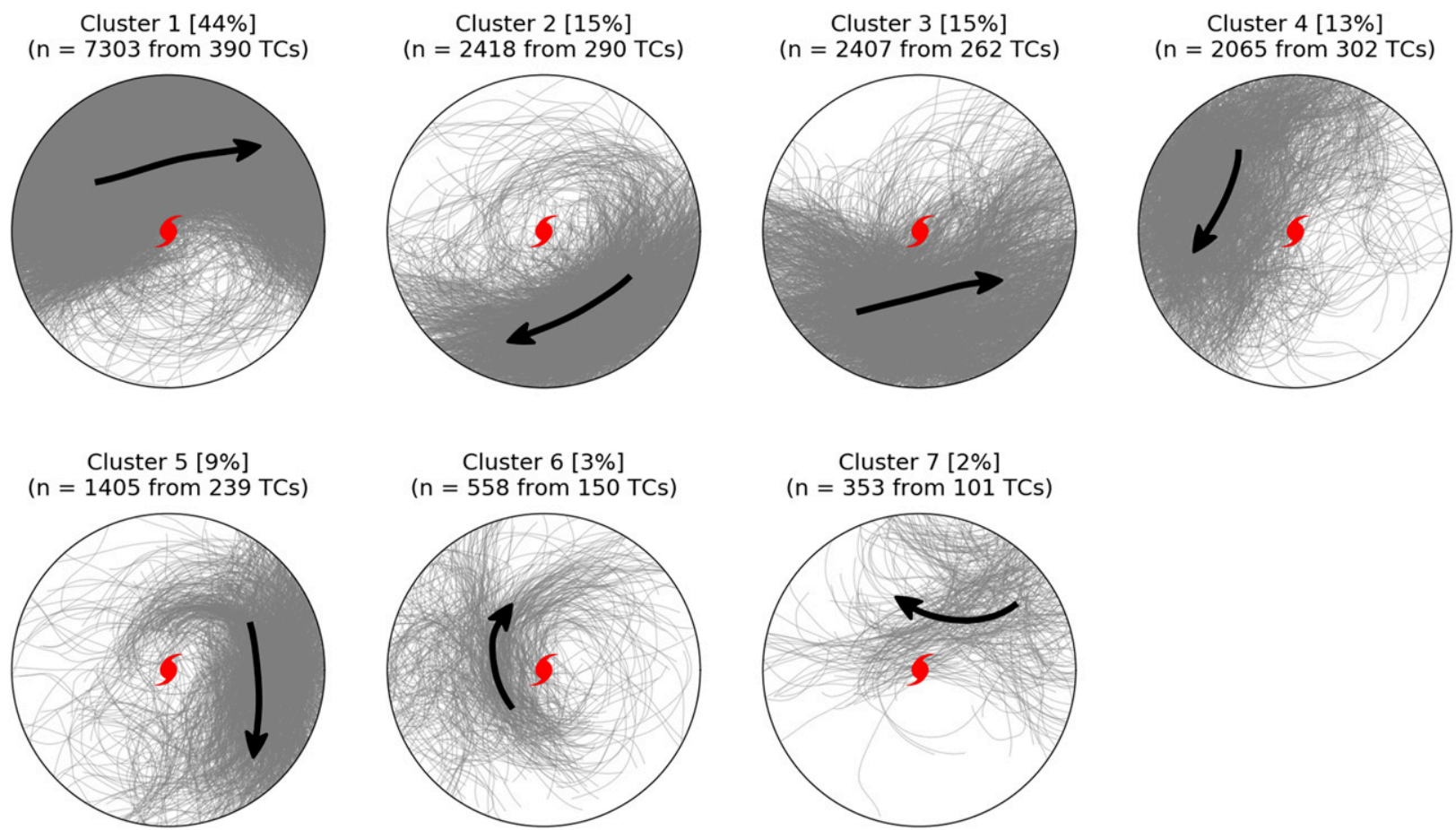

FIG. 10. Jet clusters that result from coercing the full dataset into 8 clusters. Clusters are ordered from largest to smallest, with sizes indicated. Cluster 8 has a sample size of 3 and is not shown. Each panel consists of a polar space with an outer radius of $2000 \mathrm{~km}$ centered on the TC location, denoted by the red hurricane symbol, with north pointing up. Individual jets are plotted as solid gray lines, and cluster means as black arrows. Cluster means are formed by computing the pointwise mean of all member traces after interpolating all traces to the same number of points as the longest member.

differences between each cross-group pair of individual jets are evaluated using Eq. (7).

2) The pairing arrangement is selected that minimizes the maximum difference between jet pairs. In cases where multiple arrangements have the same maximum pairwise difference, the arrangement minimizing all other pairwise differences is selected.

3) The dissimilarity measure for the two groups is then defined as the maximum pairwise difference from Eq. (7) for this optimal arrangement.

Clustering using the same UPGMA algorithm from section 5a is performed for each set of jet groups with equal sizes. As before for individual jets, the degree of clustering structure for these subsamples is not expected to be high, especially with the extra degrees of freedom introduced by grouping, which increases variance between samples. However, there is again expected to be at least some structure, and it is of interest to identify what types of multijet configurations occur and which of those are most common. The rate-distortion cost functions for groups with 1-3 jets are shown in Fig. 13. For single-jet samples, a local minimum exists at $m=18$, but it is weak relative to the cost function's value at small $m$. Nevertheless, examination of the resulting clusters suggests that the partitioning does illuminate some structure, as $88 \%$ of samples are coalesced into only 5 of the 18 clusters. This indicates that some single-jet configurations are significantly more common than others, denoting statistical modes in the dataset.

For clusters of multijet groups, an additional step to split groups into their constituents is necessary to expose the grouped structure of the clusters. This task itself necessitates clustering, since there is no way to know a priori the proper division of all group constituents. The same clustering technique used thus far can be applied to the jets from each group cluster, with the knowledge that the number of division clusters that should exist is equal to the size of the groups (e.g., jets from a cluster of two-jet groups should be divisible into two equally sized "division clusters"). For group clusters with sufficiently low distortion, the known number of division clusters should also be objectively apparent, indicated by a global minimum in the associated rate-distortion cost function at the expected value of $m$. However, this is not always the case if distortion is high. For example, the cost function for two-jet samples exhibits its strongest minimum at $m=3$ (Fig. 13b), but distortion at such a small value of $m$ is too large to unambiguously split the 
Cluster 1

( $n=7303$ from 390 TCs)

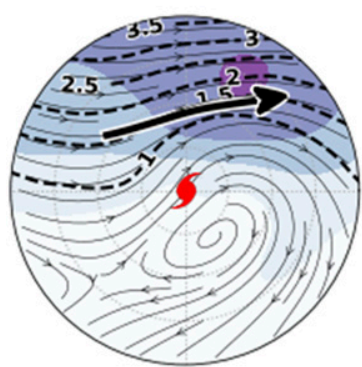

Cluster 5

( $n=1405$ from 239 TCs)

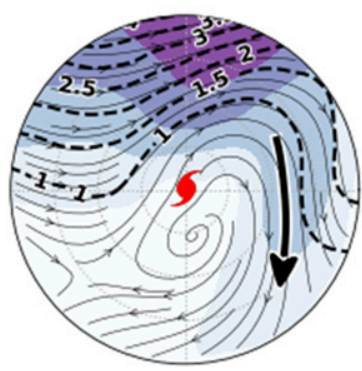

Cluster 2

( $n=2418$ from 290 TCs)

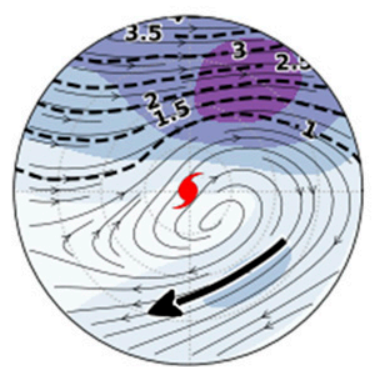

Cluster 6 ( $n=558$ from 150 TCs)

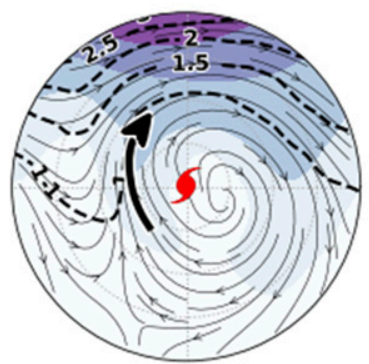

Cluster 3

( $n=2407$ from 262 TCs)

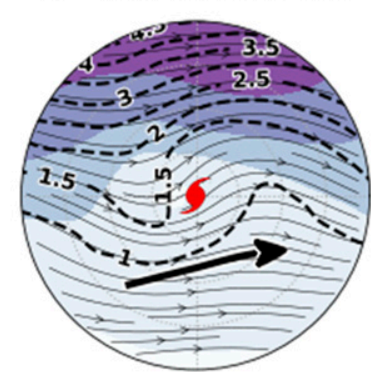

Cluster 7

( $n=353$ from 101 TCs)

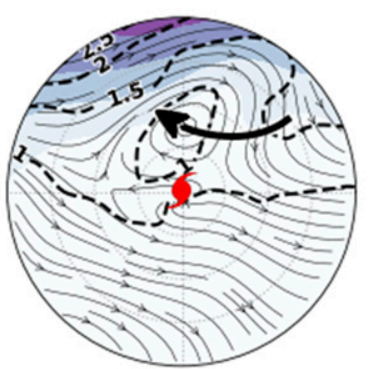

Cluster 4

( $n=2065$ from 302 TCs)
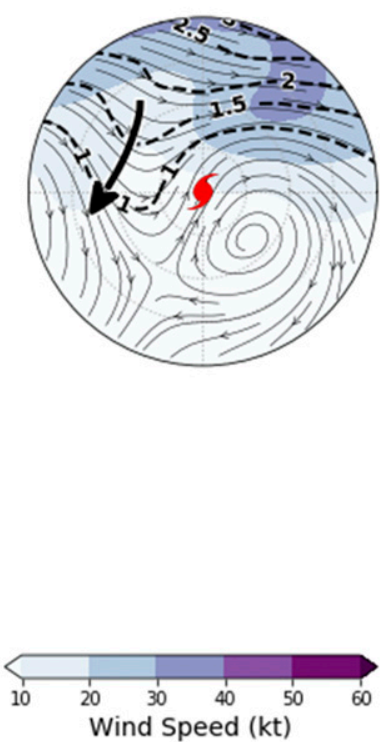

FIG. 11. Mean $200 \mathrm{hPa}$ wind (kt; shading/streamlines) and PV (dash-contoured every 0.5 PVU) for the clusters in Fig. 10. Cluster mean jet traces are plotted as black arrows, and each panel extends to a radius of $2000 \mathrm{~km}$, with north pointing up.

resulting clusters into their constituents. The solution to this problem is to reduce distortion by increasing the number of clusters until the groups are separable. A group cluster is considered separable if the value of $m$ where $C(m)$ is minimized in the domain $m \in[1,50]$ is equal to the group size and the resulting division clusters all have sizes within $1 \%$ of the group cluster's size. While increasing the number of clusters beyond the objectively best number makes the representation of the dataset less efficient, it doesn't necessarily result in a loss of structural information the way having too few clusters would, since a sufficiently small number of extra clusters often function to simply strip outliers away from the most coherent modes of the dataset.

Following this approach, for two-jet samples, the objective number of clusters is 3 , but due to high distortion, 16 clusters are required for groups within the largest modes to become separable. For three-jet samples, a weak minimum exists in the cost function at $m=18$ (Fig. 13c), but is of small magnitude relative to the global minimum at $m=1$, which is symptomatic of a nearly uniform dataset according to Kolesnikov et al. (2015). In addition, groups are not separable at that number of clusters. While some clusters become separable at $m>30$, they are few in number and not associated with large, distinct modes of the dataset. For TC samples with more than three jets, it becomes impossible for group clusters to meet the separability criteria at a reasonable number of clusters. This is likely because increasing the number of simultaneous jets within the domain decreases the average distance between individual jets, making it more difficult to split clusters into group constituents with high confidence unless distortion (and thus cluster size) is significantly reduced.

The group clusters containing at least $5 \%$ of their respective sample sets for groups of 1-3 jets are shown in Fig. 14, with group constituents differentiated by color, and objectively separable clusters indicated by bolded panels. For single-jet groups (Fig. 14a), the 5 largest of 18 clusters are shown, encompassing $88 \%$ of samples. For two-jet groups (Fig. 14b), the 4 largest of 16 clusters are shown, encompassing $86 \%$ of samples. For three-jet groups (Fig. 14c), the 5 largest of 18 clusters are shown, encompassing $86 \%$ of samples. The composite mean 200-hPa PV and wind fields associated with these clusters are shown in Fig. 15.

Single-jet groups do not require separation, and the $88 \%$ of samples shown can be broadly categorized as either environmental jets to the north of the TC (Clusters 1 and 4), outflow jets (Clusters 2 and 3), or low-tropopause cases (Cluster 5). Westerly jets poleward of the TC are by far the most common (Cluster 1 in Fig. 14a), making up $60 \%$ of single-jet groups. This indicates that when exactly one jet is present within $2000 \mathrm{~km}$, westerly poleward jets 

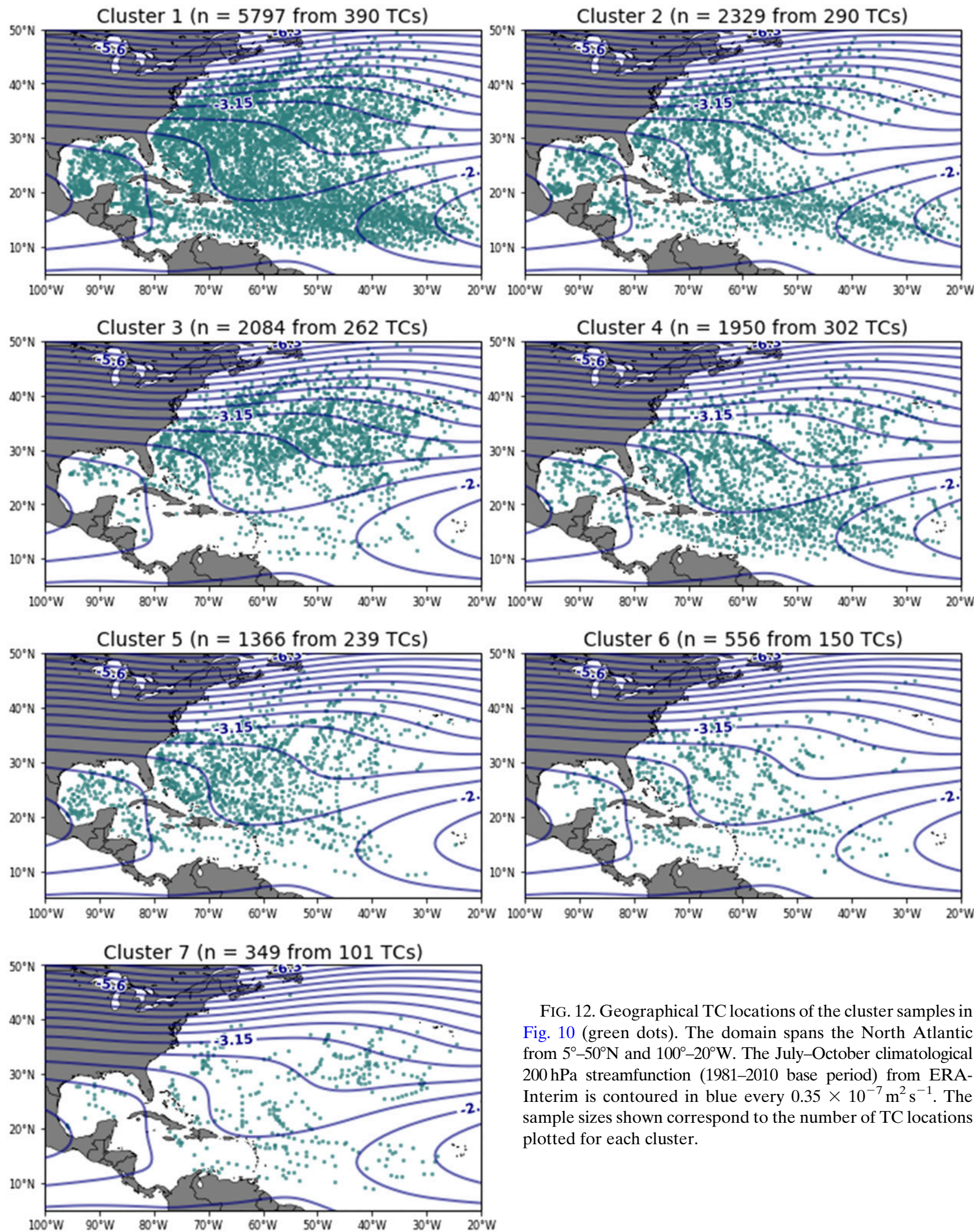

FIG. 12. Geographical TC locations of the cluster samples in Fig. 10 (green dots). The domain spans the North Atlantic from $5^{\circ}-50^{\circ} \mathrm{N}$ and $100^{\circ}-20^{\circ} \mathrm{W}$. The July-October climatological $200 \mathrm{hPa}$ streamfunction (1981-2010 base period) from ERAInterim is contoured in blue every $0.35 \times 10^{-7} \mathrm{~m}^{2} \mathrm{~s}^{-1}$. The sample sizes shown correspond to the number of TC locations plotted for each cluster. 
TABLE 1. Sample sizes, mean latitudes, and qualitative descriptions of the primary jet clusters. Sample sizes are given for the number of jets, the number of TC samples, and the number of unique TCs contained in each cluster.

\begin{tabular}{cccccc}
\hline \hline Cluster ID & Jets & TC samples & TCs & Mean latitude & Qualitative description \\
\hline 1 & 7303 & 5797 & 390 & $25.5^{\circ}$ & Poleward westerly \\
2 & 2418 & 2329 & 290 & $25.8^{\circ}$ & Equatorward outflow \\
3 & 2407 & 2084 & $31.3^{\circ}$ & Low tropopause \\
4 & 2065 & 1950 & 302 & $25.1^{\circ}$ & Anticyclonic wave break \\
5 & 1405 & 1366 & 239 & $27.2^{\circ}$ & East side outflow \\
6 & 558 & 556 & 150 & $25.3^{\circ}$ & Cyclonic wave break \\
7 & 353 & 349 & 101 & $25.5^{\circ}$ & Poleward anticyclone \\
\hline
\end{tabular}

are the most common type. The earlier results from individual clustering showed that such jets are also the most common type in general (Fig. 10).

For two-jet groups, the largest cluster (Cluster 1 in Fig. 14b) represents TC-jet configurations formed from a combination of Individual Clusters 1 and 2 in Fig. 10. As noted previously, Individual Clusters 1 and 2 have significant TC sample overlap, and two-jet Cluster 1 represents that relationship, with $81 \%$ of its TC samples found in both Individual Clusters 1 and 2. These are samples with TCs generally located beneath an elongated upper-tropospheric ridge from west-southwest to east-northeast, with peripheral jets to both the north and south. The other separable two-jet cluster (Cluster 3 in Fig. 14b) consists of jets that outline AWB events northwest of the TC. Specifically, the mean northeasterly jet to the northwest of the TC and the mean westsouthwesterly jet just north of the TC outline the positively tilted trough evident in the mean $200-\mathrm{hPa}$ flow field in Fig. 15 b (Cluster 3). This group cluster has an $82 \%$ sample intersection with Individual Cluster 4 from Fig. 10, which contains most of the northeasterly jets on the upstream flank of the aforementioned trough.

Regarding the other two (total) two-jet clusters in Fig. 14b, it should be noted that two-jet Cluster 2 is never separable for any reasonable number of division clusters. This group cluster, accounting for $29 \%$ of two-jet samples, appears to be broadly associated with TCs located between westerly jets to both the north and south, with the northern jet being strongest in the mean (Fig. 15b, Cluster 2). However, the distinction between them is unreliable. The remaining undiscussed two-jet cluster in Fig. 14b, Cluster 4, is also not objectively separable, but a clear visual division between southwesterly jets to the northwest and northerly jets to the east is evident. There is a $70 \%$ intersection of this cluster with Individual Cluster 5 from Fig. 10, which contains the northerly jets to the east of the TC. A comparison of the 200-hPa composites between the two clusters (Cluster 5 in Fig. 11 and Cluster 4 in Fig. 15b) shows that the mean states are very similar, with upper-level troughs to both the northwest and east of the TC.
Clusters of three-jet groups (Fig. 14c) are not separable with high confidence, and it is thus difficult to analyze the constituents. However, there is qualitative evidence of similarities between three-jet Clusters 1 and 2 and two-jet Clusters 1 and 3 (Fig. 14b), respectively. For three-jet Cluster 1, the same jet types as in two-jet Cluster 1 appear to exist, outlining the northern and southern flanks of an upper-tropospheric ridge, but with the addition of a third jet to the southeast,
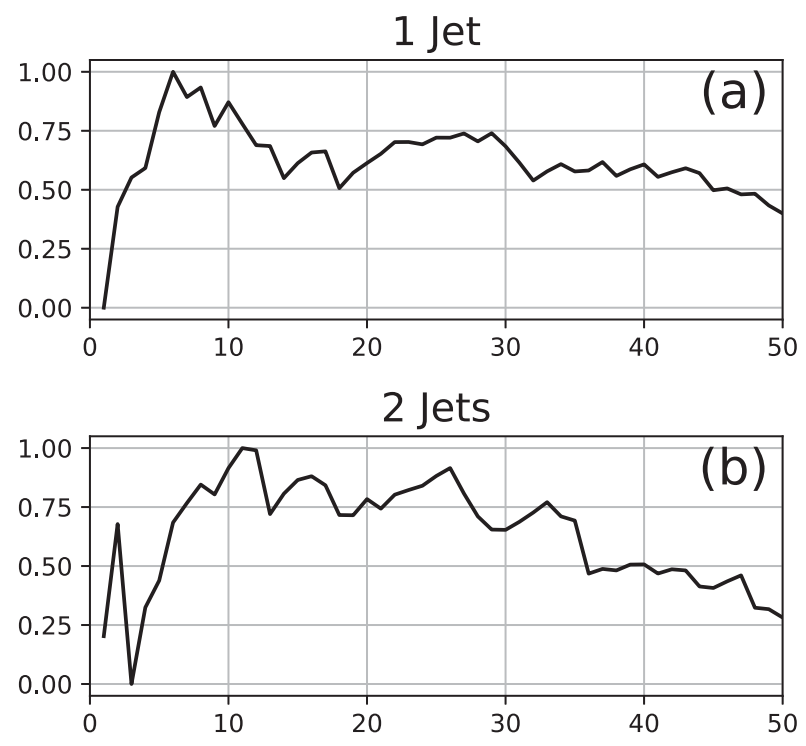

3 Jets

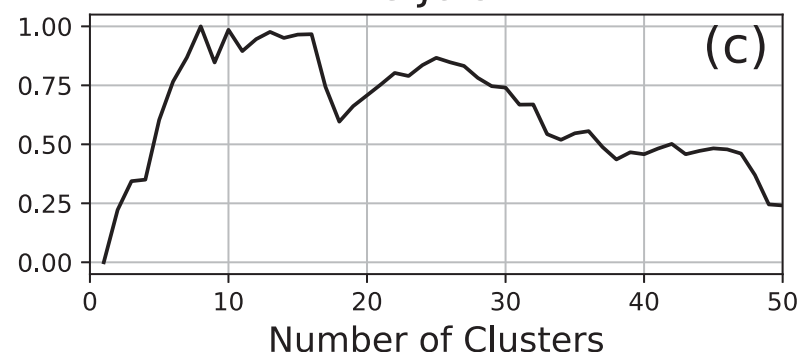

FIG. 13. Rate-distortion cost functions for jet groups from TC samples with (a) 1 jet, (b) 2 jets, and (c) 3 jets within $2000 \mathrm{~km}$ of the TC. In all cases, the cost function is formed by fitting (9) to the ratedistortion curve over the domain $m \in[1,50]$. 
(a)

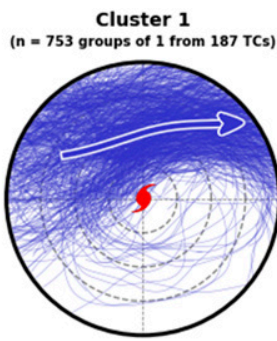

Cluster 1

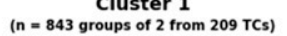

(b)

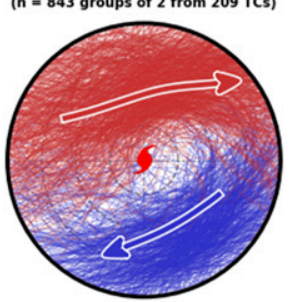

Cluster 1

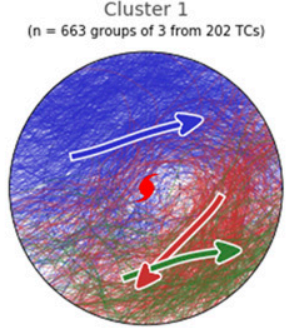

Cluster 2

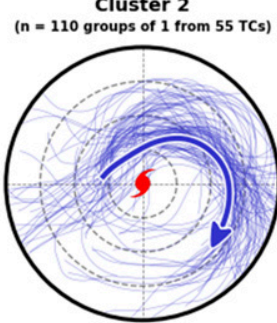

Cluster 2

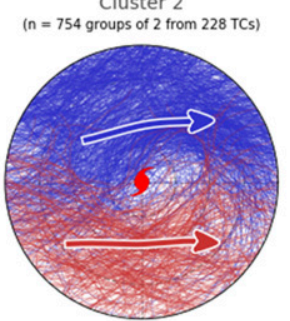

Cluster 2

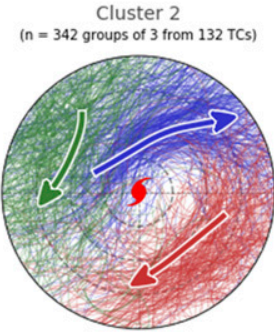

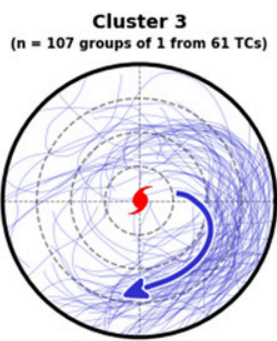

Cluster 3

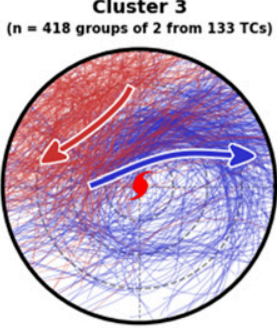

Cluster 3

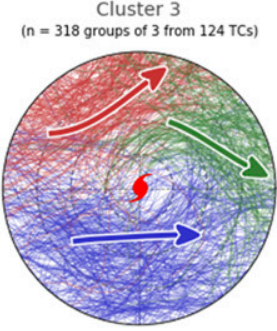

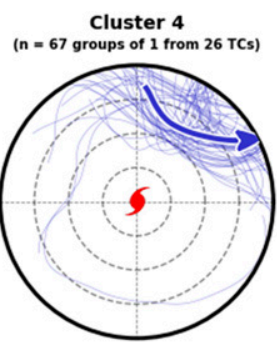

Cluster 4

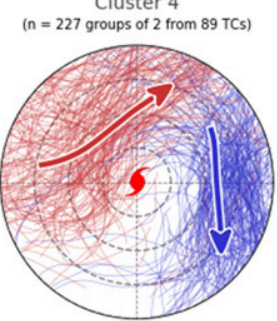

Cluster 4

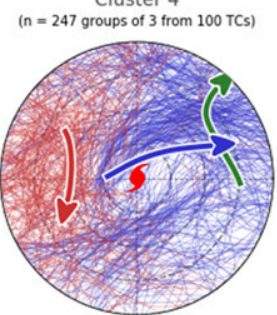

Cluster 5

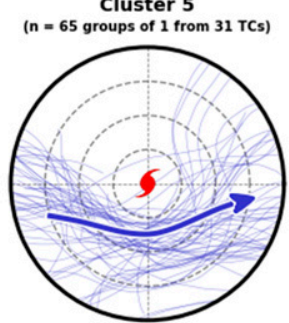

Cluster 5

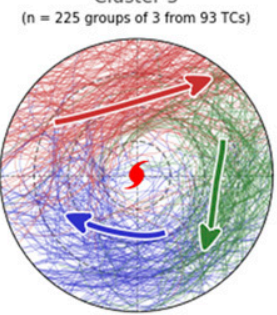

FIG. 14. Clusters of jet groups from TC samples with (a) 1 jet, (b) 2 jets, and (c) 3 jets within $2000 \mathrm{~km}$ of the TC, which is denoted by the hurricane symbol in each panel. Samples are coerced into (a) 18 clusters, (b) 16 clusters, and (c) 18 clusters. Individual jets are plotted as thin lines, organized by color to highlight group structure. For each jet in a given color group, a jet should exist in every other color group from the same TC sample, ideally. The accuracy of this color division is not guaranteed; panels with bold titles and plot borders indicate clusters which are separable with high confidence (see text). The means of each color group are plotted as thick arrows. Range rings are dotted every $500 \mathrm{~km}$, and sample sizes are indicated in the panel titles. Only clusters containing at least $5 \%$ of samples are shown. Rows (a), (b), and (c) encompass $88 \%, 86 \%$, and $86 \%$ of their respective sample sets.

oriented out of the west-southwest. These samples may correspond to TCs beneath elongated ridges with upper troughs on the southeastern flanks of the ridges, outlined by the red and green jets in Fig. 14c, Cluster 1. Some evidence for this exists in corresponding 200-hPa composite (Fig. 15c, Cluster 1), where a tongue of high PV exists southeast of the TC along the edge of the ridge, consistent with a trough at that position in the mean. Worth mentioning also is the second-largest cluster of three-jet samples, which bears a strong resemblance to the AWB pattern in two-jet Cluster 3, but with an added northeasterly jet flanking the downstream ridge to the southeast of the TC. The mean flow in Fig. 15c, Cluster 2 confirms stronger equatorward outflow in the southeast quadrant associated with these jets. The similarities between these particular two-jet and three-jet group clusters indicate that the associated flow geometries are similar, primarily differentiated by whether jets exist on both flanks of the troughs and ridges that form those geometries.
Broadly, there are two important results of the clustering analyses in this section that are relevant to the purpose of this study. The first is the identification of which TC-jet configurations are most common, and how jet axes are distributed around TCs. Jets of generally westerly orientation passing near or poleward of the TC make up $44 \%$ of all jets within $2000 \mathrm{~km}$ of Atlantic TCs, a result that is physically sensible given typical TC environments in the basin. Several other configurations of environmental jets and TC outflow jets were found, some of which occur preferentially in different regions of the Atlantic, likely due to geographic variations in the time-mean background environment. The second result of clustering is the illustration of how upper-tropospheric jets frequently trace the edges of Rossby waves and baroclinic gradients near TCs. This is unsurprising physically, but demonstrates the utility of jets for effectively and succinctly describing environmental flow near TCs. Quantitative jet datasets such as the one developed here have potential to provide unique ways of subsetting and 
(a)

1 Jet

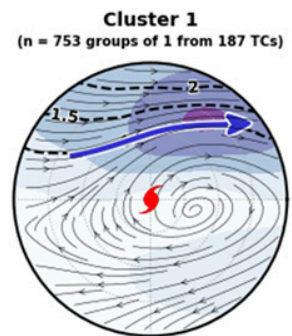

Cluster 1

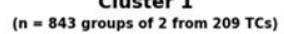

(b)

2 Jets

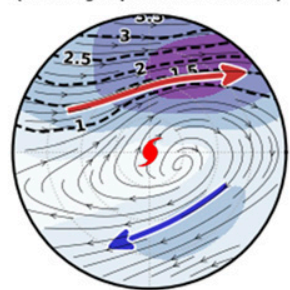

Cluster

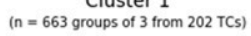

(c)

3 Jets

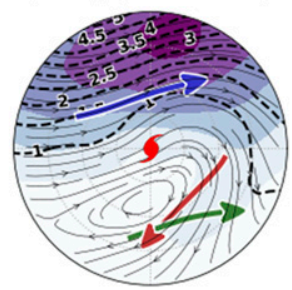

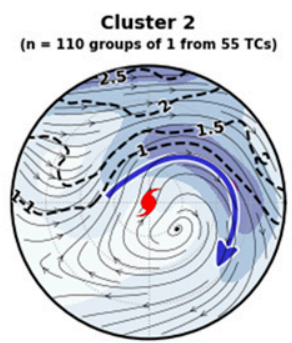

Cluster 2

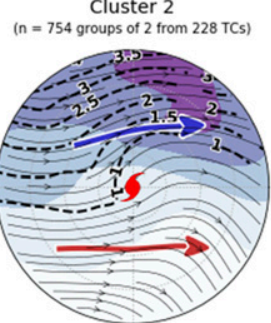

Cluster 2
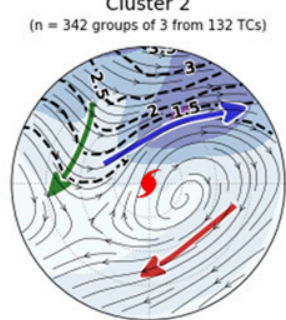

Cluster 3

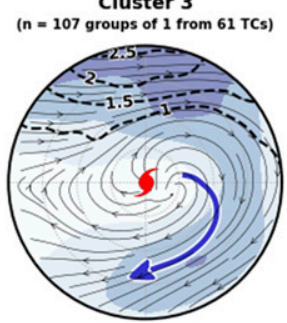

Cluster 3

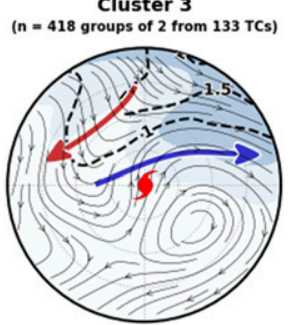

Cluster 3
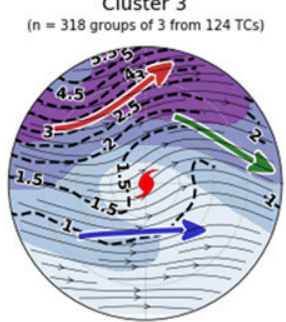

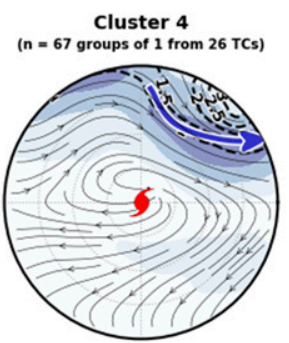

Cluster 4
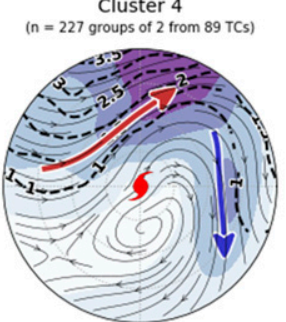

Cluster 4

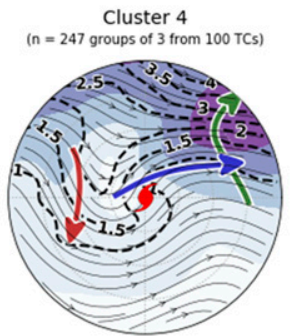

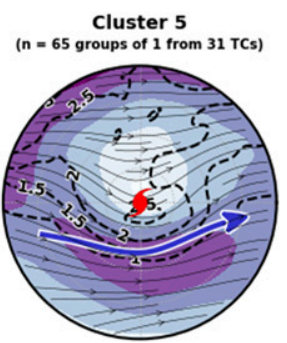

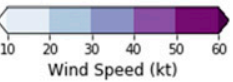

Cluster 5

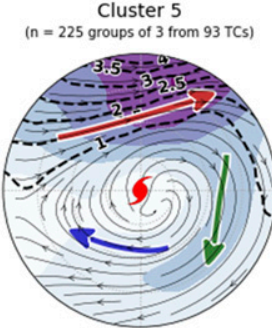

FIG. 15. Mean $200 \mathrm{hPa}$ wind (kt; shading/streamlines) and PV (dash-contoured every $0.5 \mathrm{PVU}$ ) for the jet group clusters in Fig. 14 with (a) 1 jet, (b) 2 jets, and (c) 3 jets within $2000 \mathrm{~km}$ of the TC. Mean jet traces from group constituents are plotted as thick arrows as in Fig. 14, and each panel extends to a radius of $2000 \mathrm{~km}$. Bold panels and titles denote high-confidence cluster separability as in Fig. 14.

analyzing TC flow environments and their relationships with TC behavior.

\section{Jet structure}

Other than spatial distribution, the dataset of jet axes developed here can be exploited to analyze specific aspects of TC-jet climatology. As an example, in this section we explore the climatology of jet kinematic structure near Atlantic TCs, which is related to some important physical processes based on prior work. In addition to providing information about storm environment, along-jet variability in wind speed can reveal the imprint of the nearby TC's diabatic outflow on the jet. Several studies have documented how TCs perturb the midlatitude waveguide and strengthen jets through outward advection of anticyclonic PV (e.g., Grams et al. 2011; Archambault et al. 2013; Griffin and Bosart 2014). Furthermore, as discussed in section 1, flow acceleration in jet entrance regions can also modify the TC secondary circulation in various ways. In light of these dynamical relationships identified by prior research, the following analysis examines the climatological distributions of along-jet profiles of wind magnitude, with the goal of elucidating mean jet structure near TCs in different parts of the Atlantic basin.

To assess how along-jet structure of jets interacting with TCs varies across the dataset, the environmental CPJ subset is selected as described in section 4, which broadly restricts the analysis to TC encounters with subtropical and midlatitude jets. To ensure that jets are long enough to evaluate along-jet structure upstream and downstream of the LCA, it is required that environmental CPJs span the LCA-relative distance interval $[-1000,2000] \mathrm{km}$, and that outflow CPJs span $[0,2000] \mathrm{km}$, which trims the sample sizes to 1808 and 1176, respectively. This condition also creates homogeneous samples across these distance intervals, which allows homogeneous comparisons of jet characteristics within these intervals. To accommodate the potential for points $2000 \mathrm{~km}$ downstream of the LCA to occur at radii larger than $2000 \mathrm{~km}$, the unclipped jet dataset (maximum radius of $3000 \mathrm{~km}$ instead of $2000 \mathrm{~km}$ ) is used when selecting CPJs.

A natural way to evaluate speed structure of CPJs is to align all jets at the LCA and examine the distribution of 




FIG. 16. Boxplots of environmental CPJ wind speed relative to the LCA, separated into $15^{\circ} \times 15^{\circ}$ geographical sectors. In each panel, the $y$ axis is the jet wind speed difference from the LCA wind speed (kt), and the $x$ axis is the distance downstream from the LCA (km). Boxplots are shown in 500-km increments from -1000 to $+2000 \mathrm{~km}$. The boxes encompass the interquartile range, the whiskers denote the 5th and 95th percentiles, the medians are denoted by red lines, and the means are plotted in blue. Notches in the boxes span the $95 \%$ confidence intervals about the medians, obtained through bootstrap resampling 10000 times. The numbers of contributing samples and TCs are written in the upper part of each panel in gray, and only jets spanning the entire domain $x \in[-1000,2000] \mathrm{km}$ are used, so these numbers give the sample size of all box plots in a given panel. The mean raw jet speed (kt) at the LCA is written in blue, and the mean LCA distance for each sector is written in black. Only sectors to which at least 10 TCs contribute samples are shown. Note that, as described in section 2, TC samples over land are not included.

jet magnitude in LCA-relative space. Distributions of jet speed structure are generated for different $15^{\circ} \times 15^{\circ}$ sectors of the Atlantic and shown as boxplots in Figs. 16 and 17 for environmental CPJs and outflow CPJs, respectively. The division into geographic sectors is done because the long-term mean TC kinematic environment in the Atlantic varies substantially with location, as seen in the July-October mean $200 \mathrm{hPa}$ streamfunction field in Fig. 12. The boxplots do not depict the raw jet magnitude, but rather the magnitude relative to the jet speed at the LCA. By centering speed structures in this way, changes along the jet axis (abscissa in Figs. 16 and 17) can be interpreted directly as distributions of acceleration or deceleration of the jet. Note that, here, "acceleration" refers to speed changes along the jet at a given instant in time. Mean absolute wind speeds at the LCA are provided in Figs. 16 and 17, from which the distributions of absolute jet strength can be estimated.

The environmental CPJ structures in Fig. 16 are characterized by statistically significant mean acceleration immediately downstream of the LCA extending at least $1000 \mathrm{~km}$, with the exception of the far northeastern Atlantic [panels (c) and (h)]. Acceleration near and downstream of the LCA is expected in jets nearest the TC, where strong negative PV advection by the irrotational wind occurs (Archambault et al. 2013). This influence can exert itself even on distant jets. If a jet is anticyclonic shear facing, and no other jets are present between it and the TC, the space between that jet and the TC is likely to be characterized by low PV (and thus low inertial stability). This can allow outflow to expand through the region of low inertial stability to the radius of the jet. It has been shown that radial outflow extends beyond the $2000 \mathrm{~km}$ radius in the mean (Ditchek et al. 2017), and $99.7 \%$ of CPJs considered here have a LCA closer than this distance. The mean magnitude of jet acceleration varies across the basin, with a maximum of $25-30 \mathrm{kt}$ west of $70^{\circ} \mathrm{W}$, decreasing below $20 \mathrm{kt}$ in the main development region (MDR) and northeastern Atlantic. 


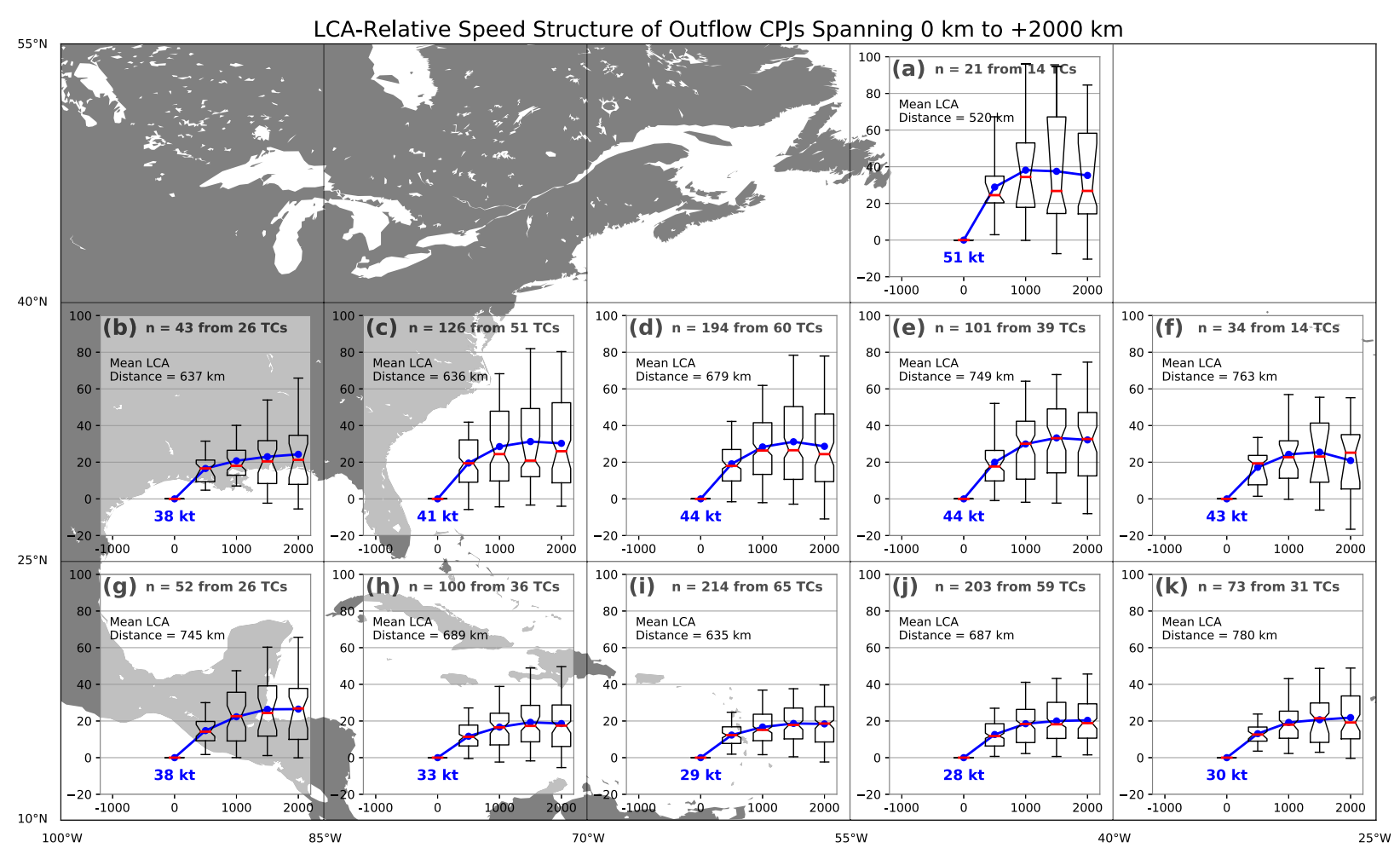

FIG. 17. As in Fig. 16, but for outflow CPJs. Only jets spanning the domain $x \in[0,2000] \mathrm{km}$ are used.

The spatial variability of jet acceleration may be understood in terms of the background state. The mean 200-hPa wind fields from samples in panels (e) and (f) and panels (g) and (h) of Fig. 16 are shown in Fig. 18, comparing the mean states between samples in the western and eastern subtropical Atlantic for TCs with environmental CPJs. The western samples (Fig. 18b) are located equatorward of the entrance region of a jet streak within the mean polar jet that passes near Newfoundland. This is an amplified version of the jet streak in the timemean climatology (Fig. 18a), possibly a result of constructive interaction between the TCs and the polar jet as radial outflow enhances acceleration in the entrance region. In contrast, eastern samples (Fig. 18c) are equatorward of the time-mean exit region of the polar jet streak. There, the TC-induced acceleration opposes the background mean deceleration, possibly causing the flatter mean acceleration profiles in the northeastern Atlantic in Fig. 16. At lower latitudes, limited along-jet acceleration observed in the MDR may be a consequence of TCs interacting with the much weaker subtropical jet, where the background PV gradient is weaker. West of $70^{\circ} \mathrm{W}$, however, samples south of $25^{\circ} \mathrm{N}$ exhibit stronger accelerations, possibly due to the aforementioned constructive interaction of TC outflow with the accelerating background mean state in the western
Atlantic, as well as closer proximity to the time-mean jet there, allowing outflow to interact with it more frequently. Mean acceleration of jets near TCs is dynamically important due to the divergent flow component and eddy momentum fluxes associated with such acceleration (Molinari and Vollaro 1989; Shi et al. 1990). Variance in entrance region amplitude near TCs across the Atlantic basin therefore implies that strong TC-jet interaction may be preferentially favored in some parts of the basin over others, but a comprehensive analysis is beyond the scope of this study.

Less variability in along-jet structure is observed for outflow CPJs (Fig. 17). Significant jet acceleration is observed downstream of the TC universally across the basin, more consistently than for environmental CPJs. This could be due to the nature of outflow CPJs, which originate within the TC outflow, and the origination of a jet implies that tightening of the environmental PV gradient (and thus jet development and acceleration) is occurring by definition. Environmental CPJs, on the other hand, approach the TC externally and parallel preexisting PV gradients upstream of the TC. They thus may or may not interact strongly with the TC outflow and accelerate downstream of their LCAs. Environmental CPJs approaching from upstream are also naturally stronger at their LCAs than outflow CPJs, which originate 

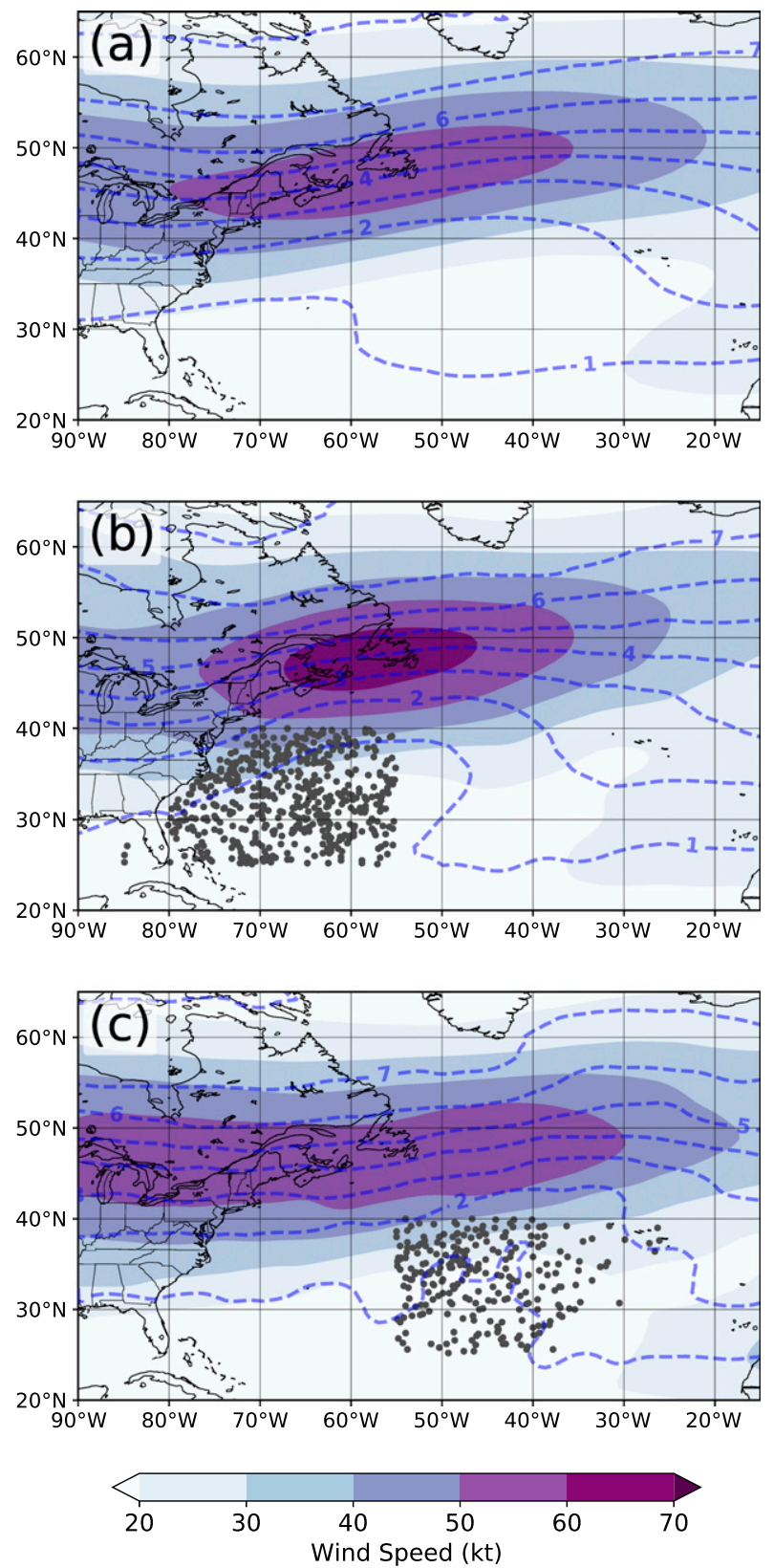

FIG. 18. Mean 200-hPa wind speed (shaded; kt) and PV (dashed contours every 1 PVU) across the North Atlantic for (a) July-October climatology, (b) samples from (e) and (f) of Fig. 16, and (c) samples from (g) and (h) of Fig. 16. In (b) and (c), TC locations from samples contributing to the mean are shown as gray dots.

at their LCAs. Comparing Figs. 16 and 17, mean jet speed at the LCA is generally $20-35 \mathrm{kt}$ faster for environmental CPJs in most geographical sectors. While outflow CPJs have more consistent acceleration across the basin, the degree of acceleration does vary, maximizing around $35 \mathrm{kt}$ in the subtropical western Atlantic [panels (e), (f), and (g) in Fig. 17], and smallest in the MDR, possibly

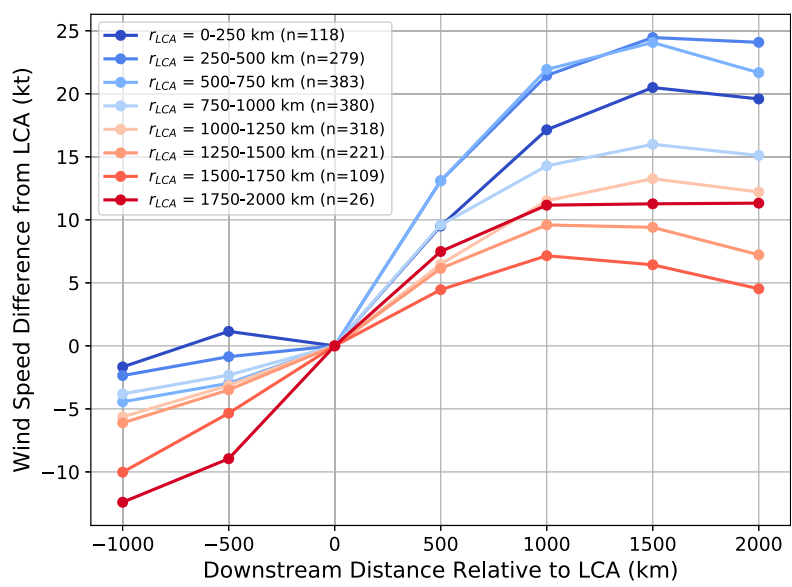

FIG. 19. Along-jet profiles of mean 200-hPa wind speed for environmental CPJs, centered about the wind speed at the LCA, for different $250-\mathrm{km}$ bins of LCA distance, identified in the legend. Profiles extend from $1000 \mathrm{~km}$ upstream to $2000 \mathrm{~km}$ downstream of the LCA in $500 \mathrm{~km}$ increments. Sample sizes of each bin are listed in the legend.

due to the same mean differences in background state as for environmental CPJs.

The magnitude and along-jet variability of flow acceleration is found to be a strong function of jet proximity. Figure 19 shows the LCA-relative wind speed distributions for different LCA distances. A clear inverse relationship exists between jet distance and acceleration downstream of the LCA, with acceleration for jets within $1000 \mathrm{~km}$ of the TC about double that of jets between 1000 and $2000 \mathrm{~km}$ from the TC (significant according to a two-sample $t$ test). This is consistent with the decaying strength of radial outflow and radiative cooling of the outflow layer at large radii, both of which would act to decrease frontogenesis, and thus jet intensification (Riemer and Jones 2010). Differences in along-jet wind speed also exist upstream of the LCA, with greater along-jet acceleration occurring in distant jets, resulting in a more continuous, smoothly increasing wind speed profile about the LCA (Fig. 19). It is not completely clear why this is the case. A speculative explanation is geometrical, recognizing the fact that the spatial rate of change of radial outflow direction is inversely proportional to radius from the TC. Outflow interacting with a jet is thus changing direction more quickly along that jet when the jet is closer to the TC, allowing a change from destructive to constructive interference between the outflow and the jet over a short distance. This could explain the more abrupt changes in jet speed observed near the LCA for smaller LCA distances. Outflow approaching more distant jets has also turned more anticyclonically by the time it reaches the jet, and may thus align constructively with the jet at points 
upstream of the LCA, leading to acceleration within that portion of the jet, something that is usually not possible for closer jets. Overall, this analysis makes clear that the distance between the TC and the jet is important to the associated jet structure, and thus to dynamical interaction between the two features, a topic that could be explored further in future work.

In the broader context of the jet dataset developed in this study, its application in this section illustrates the utility of jet axes for viewing near-TC flow from unique perspectives. Data along individual jet features facilitates quantitative analyses of TC environments that are not possible using composited flow fields. Potential applications of a TC-jet dataset such as this one are likely numerous, and extend beyond the climatological perspective elucidated here.

\section{Summary}

An objective algorithm was developed for identifying jets in 200-hPa flow, and the set of jets within $2000 \mathrm{~km}$ of Atlantic TCs during 1979-2015 was aggregated and analyzed. Jets and jet entrance regions occur most commonly poleward of TCs within the $500-1000 \mathrm{~km}$ annulus, where the confluence of TC outflow with the background PV gradient leads to acceleration of the flow and frequent generation of jets. It was shown that jets near Atlantic TCs can be optimally partitioned into seven clusters that are statistically different and correspond to recognizable TC-jet configurations. Clustering of multijet groups was much less structured, but was also able to distinguish between commonly observed flow regimes near Atlantic TCs when multiple jets are present within $2000 \mathrm{~km}$. The speed structure of westerly jets poleward of TCs was found to vary with location in the Atlantic basin, possibly due to geographical variation in the background flow poleward of TCs. Acceleration of jets downstream of their closest approach to the TC is a prominent feature across most of the basin, which is consistent with the expected response of jets to interaction with radial outflow from the TC. This objectively constructed dataset of jets could be explored more deeply in future work. It also provides novel ways of characterizing and subsetting large-scale flow near TCs, and could enable unique analyses of TC-environment interactions.

Acknowledgments. The first author was funded by Florida State University during the period of research. The second author was partially funded by NOAA Grant NA17OAR4590141. The authors are also grateful to two anonymous reviewers of this manuscript, whose helpful comments substantially improved the manuscript and provided critical feedback for future work.

\section{REFERENCES}

Ahern, K., and L. Cowan, 2018: Minimizing common errors when projecting geospatial data onto a vortex-centered space. Geophys. Res. Lett., 45, 12 032-12 039, https://doi.org/10.1029/ 2018 GL079953.

Archambault, H. M., L. F. Bosart, D. Keyser, and J. M. Cordeira, 2013: A climatological analysis of the extratropical flow response to recurving western North Pacific tropical cyclones. Mon. Wea. Rev., 141, 2325-2346, https://doi.org/10.1175/MWR-D-12-00257.1.

Bosart, L. F., W. E. Bracken, J. Molinari, C. S. Velden, and P. G. Black, 2000: Environmental influences on the rapid intensification of Hurricane Opal (1995) over the Gulf of Mexico. Mon. Wea. Rev., 128, 322-352, https://doi.org/10.1175/15200493(2000)128<0322:EIOTRI >2.0.CO;2.

Bracken, W. E., and L. F. Bosart, 2000: The role of synoptic-scale flow during tropical cyclogenesis over the North Atlantic Ocean. Mon. Wea. Rev., 128, 353-376, https://doi.org/10.1175/ 1520-0493(2000)128<0353:TROSSF $>2.0 . \mathrm{CO} ; 2$.

Corbosiero, K. L., and J. Molinari, 2002: The effects of vertical wind shear on the distribution of convection in tropical cyclones. Mon. Wea. Rev., 130, 2110-2123, https://doi.org/10.1175/ 1520-0493(2002)130<2110:TEOVWS $>2.0$.CO;2.

Cunningham, P., and D. Keyser, 2000: Analytical and numerical modelling of jet streaks: Barotropic dynamics. Quart. J. Roy. Meteor. Soc., 126, 3187-3217, https://doi.org/10.1002/qj.49712657010.

Dee, D. P., and Coauthors, 2011: The ERA-Interim reanalysis: Configuration and performance of the data assimilation system. Quart. J. Roy. Meteor. Soc., 137, 553-597, https://doi.org/ 10.1002/qj.828.

Ditchek, S. D., J. Molinari, and D. Vollaro, 2017: Tropical cyclone outflow-layer structure and balanced response to eddy forcings. J. Atmos. Sci., 74, 133-149, https://doi.org/10.1175/JASD-16-0117.1.

Eiter, T., and H. Mannila, 1994: Computing discrete Fréchet distance. Tech. Rep. CD-TR 94/64, 8 pp., http://www.kr.tuwien.ac.at/staff/ eiter/et-archive/cdtr9464.pdf.

Eliassen, A., 1951: Slow thermally or frictionally controlled meridional circulation in a circular vortex. Astrophys. Nor., 5, 19.

Fischer, M. S., B. H. Tang, and K. L. Corbosiero, 2017: Assessing the influence of upper-tropospheric troughs on tropical cyclone intensification rates after genesis. Mon. Wea. Rev., 145, 1295-1313, https://doi.org/10.1175/MWR-D-16-0275.1.

,-- , and -2019 : A climatological analysis of tropical cyclone rapid intensification in environments of uppertropospheric troughs. Mon. Wea. Rev., 147, 3693-3719, https://doi.org/10.1175/MWR-D-19-0013.1.

Grams, C. M., and Coauthors, 2011: The key role of diabatic processes in modifying the upper-tropospheric wave guide: A North Atlantic case-study. Quart. J. Roy. Meteor. Soc., 137, 2174-2193, https://doi.org/10.1002/qj.891.

Griffin, K. S., and L. F. Bosart, 2014: The extratropical transition of tropical cyclone Edisoana (1990). Mon. Wea. Rev., 142, 27722793, https://doi.org/10.1175/MWR-D-13-00282.1.

Hanley, D., J. Molinari, and D. Keyser, 2001: A composite study of the interactions between tropical cyclones and upper-tropospheric troughs. Mon. Wea. Rev., 129, 2570-2584, https://doi.org/10.1175/ 1520-0493(2001)129<2570:ACSOTI >2.0.CO;2.

Holland, G., and R. T. Merrill, 1984: On the dynamics of tropical cyclone structural changes. Quart. J. Roy. Meteor. Soc., 110, 723-745, https://doi.org/10.1002/qj.49711046510.

Klein, P. M., P. A. Harr, and R. L. Elsberry, 2002: Extratropical transition of western North Pacific tropical cyclones: Midlatitude 
and tropical cyclone contributions to reintensification. Mon. Wea. Rev., 130, 2240-2259, https://doi.org/10.1175/ 1520-0493(2002)130<2240:ETOWNP>2.0.CO;2.

Knapp, K. R., M. C. Kruk, D. H. Levinson, H. J. Diamond, and C. J. Neumann, 2010: The International Best Track Archive for Climate Stewardship (IBTrACS): Unifying tropical cyclone data. Bull. Amer. Meteor. Soc., 91, 363-376, https://doi.org/ 10.1175/2009BAMS2755.1.

Koch, P., H. Wernli, and H. C. Davies, 2006: An event-based jetstream climatology and typology. Int. J. Climatol., 26, 283-301, https://doi.org/10.1002/joc.1255.

Kolesnikov, A., E. Trichina, and T. Kauranne, 2015: Estimating the number of clusters in a numerical data set via quantization error modeling. Pattern Recognit., 48, 941-952, https://doi.org/ 10.1016/j.patcog.2014.09.017.

McTaggart-Cowan, R., J. Gyakum, and M. Yau, 2003: The influence of the downstream state on extratropical transition: Hurricane Earl (1998) case study. Mon. Wea. Rev., 131, 19101929, https://doi.org/10.1175//2589.1.

Merrill, R. T., 1988: Environmental influences on hurricane intensification. J. Atmos. Sci., 45, 1678-1687, https://doi.org/ 10.1175/1520-0469(1988)045<1678:EIOHI>2.0.CO;2.

Molinari, J., and D. Vollaro, 1989: External influences on hurricane intensity. Part I: Outflow layer eddy angular momentum fluxes. J. Atmos. Sci., 46, 1093-1105, https://doi.org/10.1175/ 1520-0469(1989)046<1093:EIOHIP>2.0.CO;2.

, and _ 1990: External influences on hurricane intensity. Part II: Vertical structure and response of the hurricane vortex. J. Atmos. Sci., 47, 1902-1918, https://doi.org/10.1175/15200469(1990)047<1902:EIOHIP>2.0.CO;2.

— S. Skubis, D. Vollaro, F. Alsheimer, and H. E. Willoughby, 1998: Potential vorticity analysis of tropical cyclone intensification. J. Atmos. Sci., 55, 2632-2644, https://doi.org/10.1175/ 1520-0469(1998)055<2632:PVAOTC $>2.0 . C O ; 2$.

Moore, J. T., and G. E. Vanknowe, 1992: The effect of jet-streak curvature on kinematic fields. Mon. Wea. Rev., 120, 2429-2441, https://doi.org/10.1175/1520-0493(1992)120<2429:TEOJSC > 2.0.CO;2.

Park, K., X. Zou, and G. Li, 2009: A numerical study on rapid intensification of Hurricane Charley (2004) near landfall. Front. Earth Sci. China, 3, 457-470, https://doi.org/10.1007/s11707009-0048-y.

Pfeffer, R. L., and M. Challa, 1981: A numerical study of the role of eddy fluxes of momentum in the development of Atlantic hurricanes. J. Atmos. Sci., 38, 2393-2398, https://doi.org/ 10.1175/1520-0469(1981)038<2393:ANSOTR > 2.0.CO;2.

Pyle, M. E., D. Keyser, and L. F. Bosart, 2004: A diagnostic study of jet streaks: Kinematic signatures and relationship to coherent tropopause disturbances. Mon. Wea. Rev., 132, 297-319, https://doi.org/10.1175/1520-0493(2004)132<0297:ADSOJS > 2.0.CO;2.

Rappin, E. D., M. C. Morgan, and G. J. Tripoli, 2011: The impact of outflow environment on tropical cyclone intensification and structure. J. Atmos. Sci., 68, 177-194, https://doi.org/10.1175/ 2009JAS2970.1.

Riemer, M., and S. C. Jones, 2010: The downstream impact of tropical cyclones on a developing baroclinic wave in idealized scenarios of extratropical transition. Quart. J. Roy. Meteor Soc., 136, 617-637, https://doi.org/10.1002/QJ.605.

Shi, J.-J., S. Wei-Jen Chang, and S. Raman, 1990: A numerical study of the outflow layer of tropical cyclones. Mon. Wea. Rev., 118, 2042-2055, https://doi.org/10.1175/1520-0493(1990)118<2042: ANSOTO $>2.0 . \mathrm{CO} ; 2$.

Shi, J. J., S. Chang, and S. Raman, 1997: Interaction between Hurricane Florence (1988) and an upper-tropospheric westerly trough. J. Atmos. Sci., 54, 1231-1247, https://doi.org/ 10.1175/1520-0469(1997)054<1231:IBHFAA > 2.0.CO;2.

Sneath, P. H., and R. R. Sokal, 1973: Numerical Taxonomy: The Principles and Practice of Numerical Classification. W. H. Freeman, 573 pp.

Snyder, J. P., 1987: Map projections-A working manual. Vol. 1395, U.S. Government Printing Office, 383 pp.

Sokal, R. R., and C. D. Michener, 1958: A statistical method for evaluating systematic relationship. Univ. Kans. Sci. Bull., 28, $1409-1438$

Spensberger, C., T. Spengler, and C. Li, 2017: Upper-tropospheric jet axis detection and application to the boreal winter 2013/14 . Mon. Wea. Rev., 145, 2363-2374, https://doi.org/10.1175/MWRD-16-0467.1.

Uccellini, L. W., and P. J. Kocin, 1987: The interaction of jet streak circulations during heavy snow events along the east coast of the United States. Wea. Forecasting, 2, 289-308, https://doi.org/ 10.1175/1520-0434(1987)002<0289:TIOJSC $>2.0 . C O ; 2$.

Wu, C.-C., and H.-J. Cheng, 1999: An observational study of environmental influences on the intensity changes of Typhoons Flo (1990) and Gene (1990). Mon. Wea. Rev., 127, 3003-3031, https://doi.org/10.1175/1520-0493(1999)127<3003:AOSOEI> 2.0.CO;2. 